\title{
Absolute Stability With a Generalized Sector Condition
}

\author{
Tingshu Hu, Senior Member, IEEE, Bin Huang, Student Member, IEEE, and Zongli Lin, Senior Member, IEEE
}

\begin{abstract}
This paper generalizes the linear sector in the classical absolute stability theory to a sector bounded by concave/convex functions. This generalization allows more flexible or more specific description of the nonlinearity and will thus reduce the conservatism in the estimation of the domain of attraction. We introduce the notions of generalized sector and absolute contractive invariance for estimating the domain of attraction of the origin. Necessary and sufficient conditions are identified under which an ellipsoid is absolutely contractively invariant. In the case that the sector is bounded by piecewise linear concave/convex functions, these conditions can be exactly stated as linear matrix inequalities. Moreover, if we have a set of absolutely contractively invariant (ACI) ellipsoids, then their convex hull is also ACI and inside the domain of attraction. We also present optimization technique to maximize the absolutely contractively invariant ellipsoids for the purpose of estimating the domain of attraction. The effectiveness of the proposed method is illustrated with examples.
\end{abstract}

Index Terms-Absolute contractive invariance (ACI), absolute stability, concave function, convex function, generalized sector.

\section{INTRODUCTION}

$\mathbf{C}$ ONSIDER the Lur'e system in Fig. 1, where $A \in \mathbf{R}^{n \times n}$, $B \in \mathbf{R}^{n \times 1}, F \in \mathbf{R}^{1 \times n}$ and $v=\psi(u, t):(\mathbf{R}, \mathbf{R}) \mapsto \mathbf{R}$ belongs to a class of sector bounded nonlinear functions. The absolute stability of the Lur'e system in Fig. 1 is a classical problem in control theory. It has been addressed extensively in the nonlinear systems and control literature (see, e.g., [1], [12], [18], [24], [26], [27], [29], [31], [32], and the references therein), and is still attracting tremendous attention (see [2], [5], [6], [9], [10], [21], [22], [25], and [28] for a sample of recent literature). The traditional tools for absolute stability analysis include circle criterion and Popov criterion. With the advancement of robust control theory in the 1980s and 1990s, some close relationship between absolute stability and robustness was established in [5] and robustness analysis tools were applied to deal with absolute stability problems (see, e.g., [5], [9], [18], [25]). In [18], an absolute stability criterion stemming from the vertex criterion in robustness analysis was presented along with circle and Popov criteria. (We will call the first one vertex criterion in this paper.) Among these tools, circle criterion appears to be the most popular, but as was implied in [18], circle criterion is more conservative than the vertex criterion. More importantly,

Manuscript received November 5, 2002; revised October 7, 2003. Recommended by Associate Editor R. Freeman. This work was supported in part by the National Science Foundation under Grant CMS-0324329.

The authors are with the Department of Electrical and Computer Engineering, University of Virginia, Charlottesville, VA 22904-4743 USA (e-mail: th7f@ virginia.edu; bh4h@virginia.edu; zl5y@ virginia.edu).

Digital Object Identifier 10.1109/TAC.2004.825657

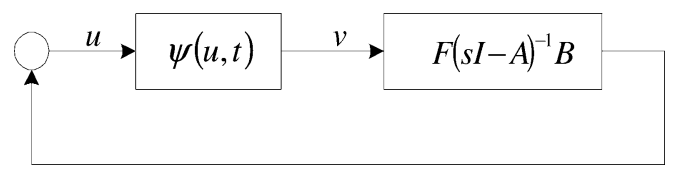

Fig. 1. System with a nonlinear component.

with the linear matrix ineqaulity (LMI) optimization techniques [4], the vertex criterion is easier to handle.

A major trend in the development of absolute stability theory is the study of absolute stability within a finite region. Since global absolute stability does not generally hold, the study of absolute stability within a finite region is also of great significance and has been studied extensively (e.g., in [6], [10], [18], and [29]-[31]). In the case where global absolute stability does not hold, we need to restrict our attention to a finite region in the state space, where a sector that is narrower than the global sector can be used to bound the nonlinear function $\psi(u, t)$. Fig. 2 plots a sector between two straight lines $v=k_{1} u$ and $v=k_{2} u$. This sector is a global bound for one of the nonlinear functions but is only a local bound for the other one, which can only be globally bounded by $v=k_{1} u$ and $v=0$. In the finite region, a guaranteed domain of attraction can be then obtained by using some invariant level set of a quadratic or Lur'e type Lyapunov function. In this paper, we will study the absolute stability in a finite region.

A very common type of nonlinearity is the standard saturation function $v=\operatorname{sat}(u)$ and saturation like functions such as $v=\tan ^{-1}(u)$ (plotted as one of the functions in Fig. 2). Stability analysis and design for systems with saturation nonlinearities has received tremendous attention in recent years (see [3], [13], [20], and the references therein). One of the fundamental problems is the characterization of the domain of attraction. While analytical characterization of the domain of attraction has been attempted and is believed to be extremely hard except for some special cases (see, e.g., [17]), most of the literature is dedicated to obtaining an estimate of the domain of attraction with reduced conservatism or to enlarging some invariant set inside the domain of attraction by appropriately designing the feedback gain. Along this direction, the classical absolute stability analysis tools such as circle criterion, Popov criterion and the vertex criterion have been used to obtain an estimate of the domain of attraction (see, e.g., [7], [8], [11], [18], [19], [23], and [30]). Typically, the domain of attraction is estimated by using invariant ellipsoids.

Recently, we developed a new sufficient condition for an ellipsoid to be invariant under saturation nonlinearities in [16] (see also [13]). It was shown that this condition is less conservative than the existing conditions resulting from the circle criterion 


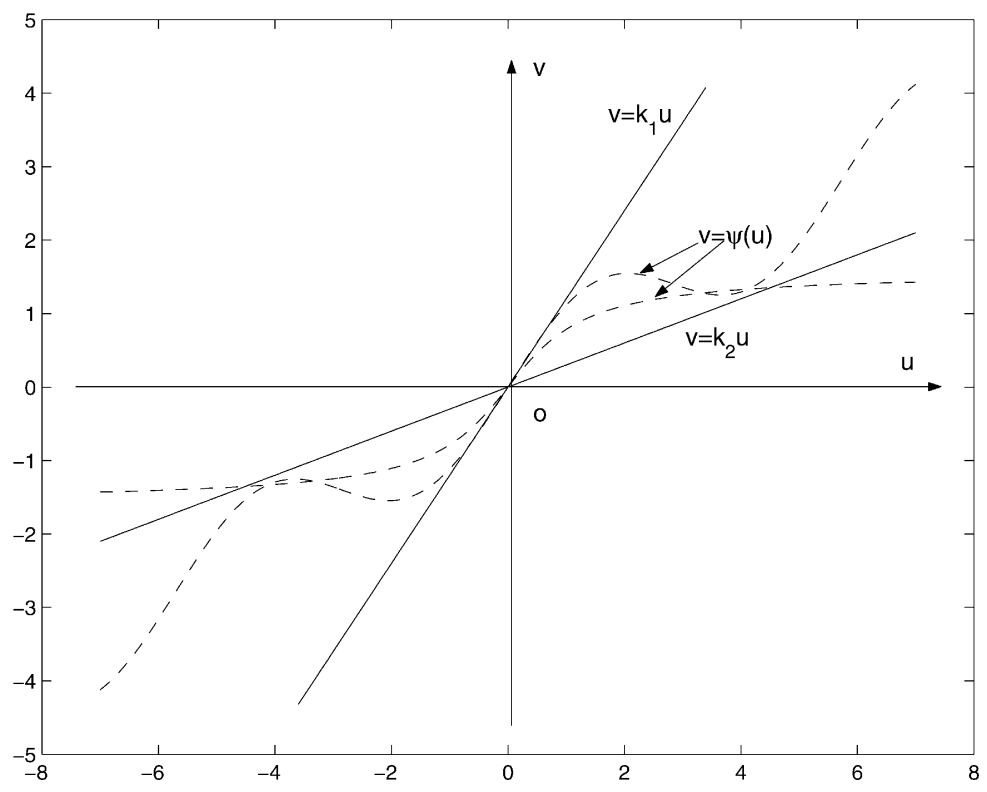

Fig. 2. Classical linear sector.

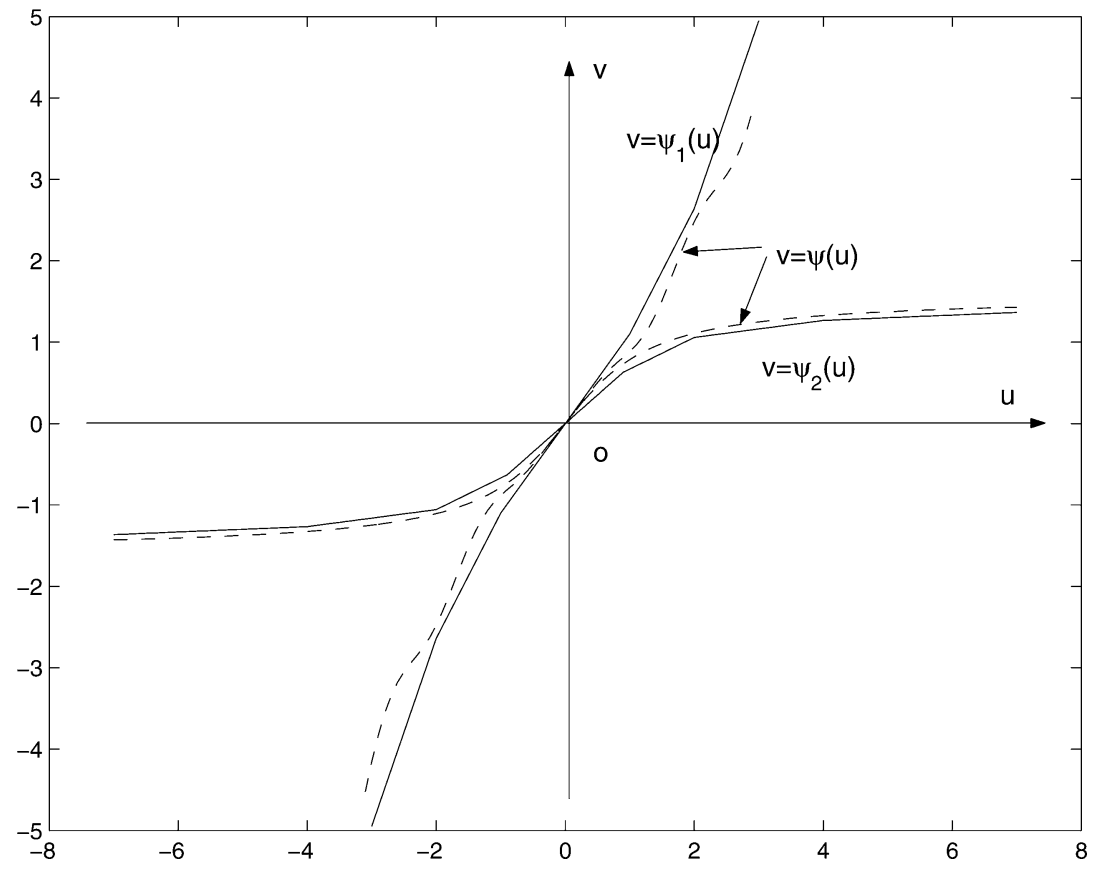

Fig. 3. Generalized sector.

or the vertex criterion. The most important feature of this new condition is that it can be expressed as LMIs in terms of all the varying parameters and, hence, can easily be used for controller synthesis. A recent discovery makes this condition even more attractive. In [14], we showed that, for single input systems, this condition is also necessary, thus, the largest invariant ellipsoid can be exactly obtained by solving an LMI optimization problem.

Encouraged by our recent stability analysis results on saturation nonlinearity, we attempt to address more general class of nonlinearities, possibly time varying and with uncertainty. We will formulate a problem that is a generalization of the classical absolute stability problem. The motivation is as follows. In the classical absolute stability problem, the nonlinear func- tion is within a sector which is bounded by two linear functions. As we can see from Fig. 2, the sector is not a tight bound for such functions as $v=\tan ^{-1}(u)$ (the difference between $v=\tan ^{-1}(u)$ and $v=k_{2} u$ is considerably large between the two intersections). This accounts for the conservatism in determining the largest invariant ellipsoid by using the circle criterion or the vertex criterion (see [16]). In view of this, we would like to introduce a generalized sector bound as plotted in Fig. 3. In Fig. 3, the two solid curves are $v=\psi_{1}(u)$ and $v=\psi_{2}(u)$. They are piecewise linear odd symmetric functions. $\psi_{1}(u)$ is convex for $u>0$ and $\psi_{2}(u)$ is concave for $u>0$. The saturation like function in dashed curve is $v=\tan ^{-1}(u)$. In this paper, we will consider the absolute stability problem for the system in Fig. 1 with $\psi(u, t)$ lying between two piecewise linear functions 


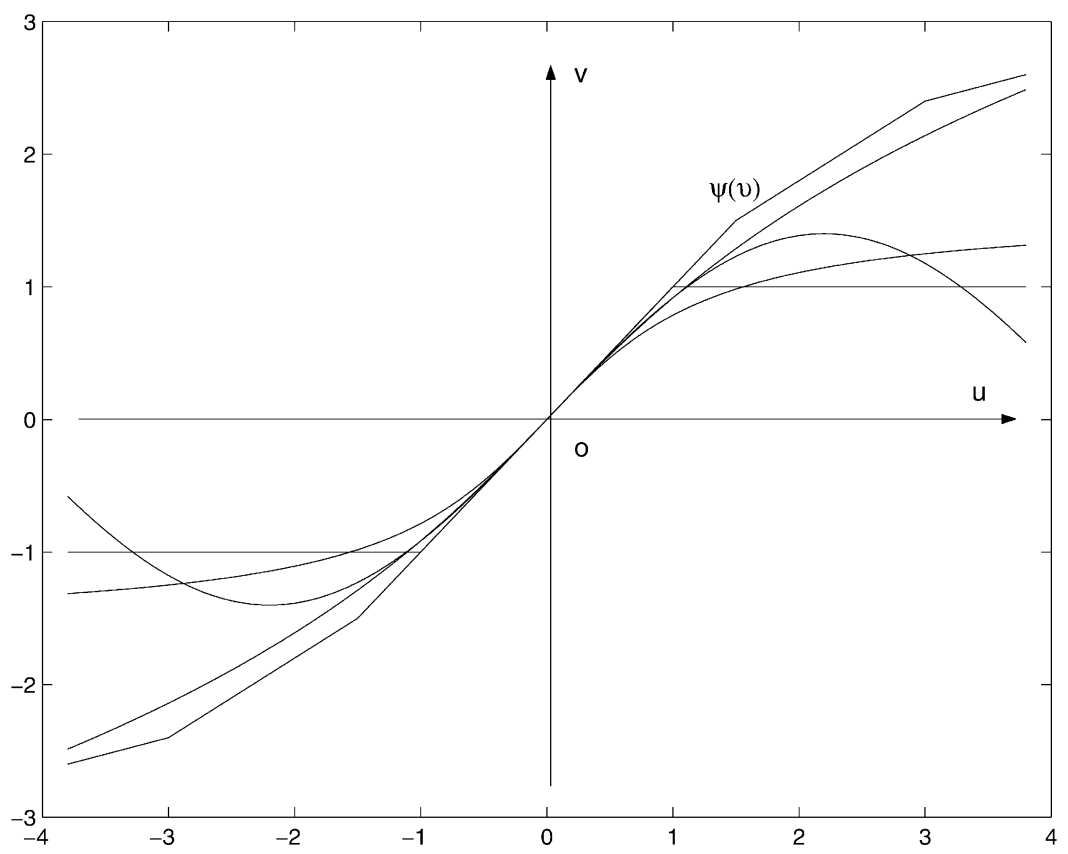

Fig. 4. Class of concave functions.

$\psi_{1}(u)$ and $\psi_{2}(u)$. We call the region between $\psi_{1}(u)$ and $\psi_{2}(u)$ the generalized sector. In Fig. 3, the function $\psi_{1}(u)$ is convex and $\psi_{2}(u)$ is concave for $u>0$. We may also use two functions that are both concave (or both convex) for $u>0$ to define a generalized sector. For example, the saturation like function in Fig. 3 would be more tightly bounded by two concave piecewise linear functions. We will establish the necessary and sufficient condition for the contractive invariance of an ellipsoid for all the possible nonlinear functions $\psi(u, t)$ in a generalized sector. An ellipsoid that is contractively invariant for every nonlinear function $\psi(u, t)$ in the generalized sector is said to be absolutely contractively invariant (ACI). By using the result in [15], we will see that if we have a set of ACI ellipsoids, then their convex hull is also ACI.

This paper is organized as follows. In Section II, we define a class of concave (or convex) nonlinear functions and introduce the notions of generalized sector and absolute contractive invariance. We also recall a recent result on saturation nonlinearity which will be very useful in this paper. In Section III, we derive necessary and sufficient conditions for an ellipsoid to be invariant under a piecewise linear function with only one bend, a piecewise linear function with multiple bends and a general concave (or convex) function. In Section IV, we show that under convex or concave nonlinearities, the convex hull of a set of invariant ellipsoids is also an invariant set. In Section V, we present the necessary and sufficient condition for an ellipsoid to be ACI and show that the convex hull of a set of ACI ellipsoids is also ACI and inside the domain of attraction. In Section VI, we present methods for estimating the domain of attraction with an LMI technique. Section VII concludes this paper.

Notation: For two intergers $k_{1}, k_{2}, k_{1}<k_{2}$, we denote $I\left[k_{1}, k_{2}\right]=\left\{k_{1}, k_{1}+1, \ldots, k_{2}\right\}$. We use $\operatorname{sat}(\cdot)$ to denote the standard saturation function, i.e., $\operatorname{sat}(u)=\operatorname{sign}(u) \min \{1,|u|\}$. For a set of vectors $x_{1}, x_{2}, \ldots, x_{N} \in \mathbf{R}^{n}$, we use $\operatorname{co}\left\{x_{1}, x_{2}, \ldots, x_{N}\right\}$ to denote the convex hull of these vectors, i.e.,

$$
\operatorname{co}\left\{x_{1}, x_{2}, \ldots, x_{N}\right\}:=\left\{\sum_{i=1}^{N} \gamma_{i} x_{i}: \sum_{i=1}^{N} \gamma_{i}=1, \gamma_{i} \geq 0\right\} .
$$

For a row vector $F \in \mathbf{R}^{1 \times n}$, denote

$$
\mathcal{L}(F):=\left\{x \in \mathbf{R}^{n}:|F x| \leq 1\right\} .
$$

Let $P=P^{T} \in \mathbf{R}^{n \times n}$ be a positive-definite matrix. For a positive number $\rho$, denote

$$
\mathcal{E}(P, \rho):=\left\{x \in \mathbf{R}^{n}: x^{T} P x \leq \rho\right\} .
$$

For simplicity, we use $\mathcal{E}(P)$ to denote $\mathcal{E}(P, 1)$.

\section{Problem Statement AND Preliminaries}

\section{A. Concave Functions and Convex Functions}

The generalized sector in this paper will be defined in terms of a class of piecewise linear symmetric functions. We first give formal definition of some functions that we will use to define the boundary of the generalized sector.

Given a scalar function $v=\psi(u)$. Assume that

1) $\psi(u)$ is continuous, piecewise differentiable, $\psi(0)=0$ and $\left.(d \psi / d u)\right|_{u=0}>0$

2) $\psi(u)$ is odd symmetric, i.e., $\psi(-u)=-\psi(u)$.

A function $\psi(u)$ satisfying the above assumption is said to be concave if it is concave for $u>0$. That is, for any $u_{1}, u_{2}>0$

$\psi\left(\gamma u_{1}+(1-\gamma) u_{2}\right) \geq \gamma \psi\left(u_{1}\right)+(1-\gamma) \psi\left(u_{2}\right) \quad \forall \gamma \in[0,1]$.

A function $\psi(u)$ satisfying the above assumption is said to be convex if it is convex for $u>0$. That is, for any $u_{1}, u_{2}>0$

$\psi\left(\gamma u_{1}+(1-\gamma) u_{2}\right) \leq \gamma \psi\left(u_{1}\right)+(1-\gamma) \psi\left(u_{2}\right) \quad \forall \gamma \in[0,1]$. 


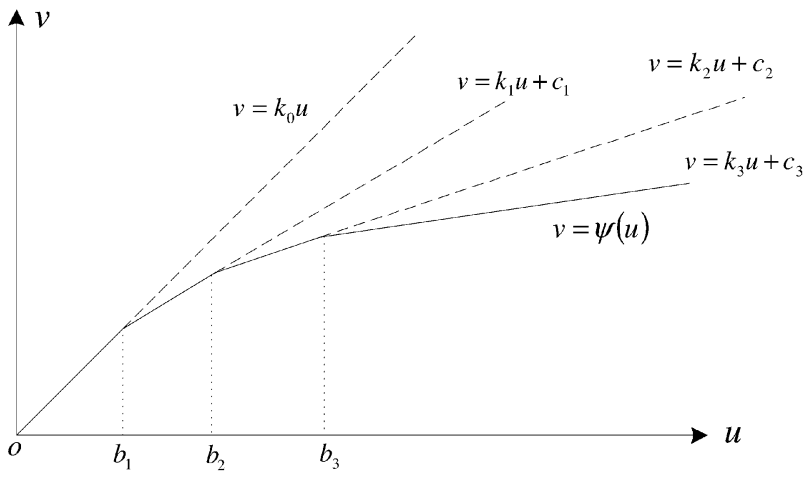

Fig. 5. Piecewise linear function with three bends.

These definitions are made for simplicity. It should be understood by odd symmetry that a concave function is convex for $u<0$ and a convex function is concave for $u<0$. Fig. 4 illustrates a few concave functions.

Here, is a simple fact about concave and convex functions.

Fact 1: Let $\psi(u)$ be a concave (convex) function. Given $u_{0}>0$, if we draw a straight line that is tangential to $\psi(u)$ at $\left(u_{0}, \psi\left(u_{0}\right)\right)$, then the straight line is above (below) $\psi(u)$ for all $u>0$.

We will first study the class of piecewise linear functions

$$
\psi(u)= \begin{cases}k_{0} u, & \text { if } u \in\left[0, b_{1}\right] \\ k_{1} u+c_{1}, & \text { if } u \in\left[b_{1}, b_{2}\right] \\ \vdots & \\ k_{N} u+c_{N}, & \text { if } u \in\left(b_{N}, \infty\right)\end{cases}
$$

where $0<b_{1}<b_{2}<\cdots<b_{N}$. The values of $\psi(u)$ for $u<$ 0 can be determined by odd symmetry, for example, $\psi(u)=$ $k_{1} u-c_{1}$ for $u \in\left[-b_{2},-b_{1}\right)$. It is easy to see that if $\psi(u)$ is concave, then $k_{0}>k_{1}>k_{2}>\cdots>k_{N}$ and $0<c_{1}<c_{2}<$ $\cdots<c_{N}$. The last slope $k_{N}$ can be positive, negative or zero. In the case that $k_{N}=0, \psi(u)$ is a saturation like function with a saturation bound $c_{N}$. If $\psi(u)$ is convex, then $0<k_{0}<k_{1}<$ $k_{2}<\cdots<k_{N}<\infty$ and $0>c_{1}>c_{2}>\cdots>c_{N}$. We note that $c_{1}, c_{2}, \ldots, c_{N}$ can be determined from $b_{1}, b_{2}, \ldots, b_{N}$ by the continuity of the function, and vise versa. Fig. 5 plots a piecewise linear concave function with three bends.

The simplest piecewise linear function is

$$
\psi(u)= \begin{cases}k_{0} u, & \text { if } u \in\left[0, b_{1}\right] \\ k_{1} u+c_{1}, & \text { if } u \in\left(b_{1}, \infty\right) .\end{cases}
$$

If $\psi(u)$ is concave, then $0<k_{1}<k_{0}, c_{1}>0$ and $b_{1}=$ $c_{1} /\left(k_{0}-k_{1}\right)$. If $\psi(u)$ is convex, then $k_{1}>k_{0}>0, c_{1}<0$ and $b_{1}=c_{1} /\left(k_{0}-k_{1}\right)$. Fig. 6 plots a concave $\psi(u)$. If $k_{0}=$ $1, k_{1}=0$ and $b_{1}=1$, then $\psi(u)$ is the standard saturation function $\operatorname{sat}(u)$. The class of piecewise linear functions with one bend is very important. All the results for general concave or convex functions will be based on those for this class of simple piecewise linear functions.

\section{B. The Generalized Sector and Absolute Stability}

The state-space description of the system in Fig. 1 is

$$
\dot{x}=A x+B \psi(F x, t) .
$$

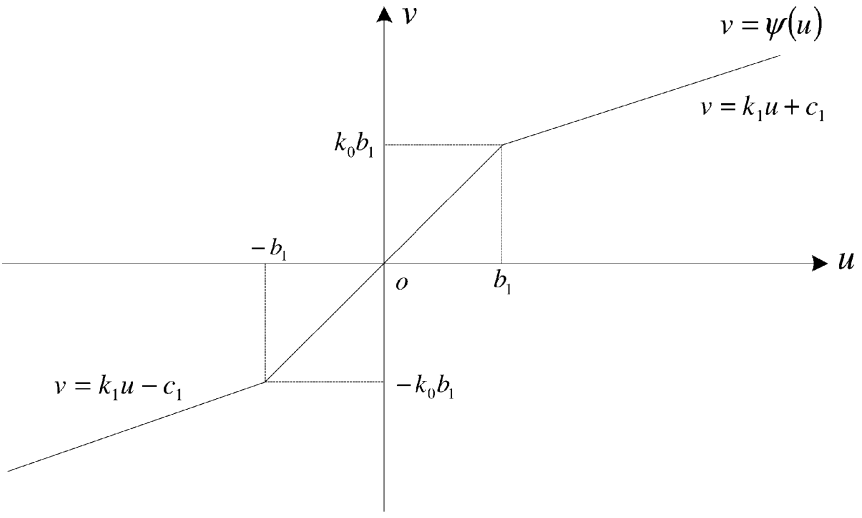

Fig. 6. Piecewise linear function with one bend.

A traditional way to estimate the domain of attraction of the origin is to use invariant sets, among which invariant ellipsoids are the most popular.

Let $V(x)$ be a differentiable positive-definite function. Given a positive number $\rho$, a level set of $V(x)$ is

$$
L_{V}(\rho):=\left\{x \in \mathbf{R}^{n}: V(x) \leq \rho\right\} .
$$

The level set $L_{V}(\rho)$ is said to be contractively invariant for (2) if

$$
\dot{V}=\left(\frac{\partial V}{\partial x}\right)^{T}(A x+B \psi(F x, t))<0
$$

for all $x \in L_{V}(\rho) \backslash\{0\}$ and $t \in \mathbf{R}$. Clearly, if $L_{V}(\rho)$ is contractively invariant, then it is inside the domain of attraction. If $V(x)$ is the quadratic function $x^{T} P x$, then $L_{V}(\rho)=\mathcal{E}(P, \rho)$.

In the aforementioned definition of contractive invariance, the nonlinear function $\psi(u, t)$ is assumed to be known. In practice, there always exists some degree of uncertainty about a nonlinear component. In view of this, we would like to study the invariance of a level set for a class of nonlinear functions, for example, a class of $\psi(u, t) \in \operatorname{co}\left\{\psi_{1}(u), \psi_{2}(u)\right\}$, where $\psi_{1}(u)$ and $\psi_{2}(u)$ are known functions. On the other hand, some nonlinear function $\psi(u, t)$ could be very complicated and we would like to bound it with simpler functions $\psi_{1}(u)$ and $\psi_{2}(u)$. These problems arise from the same situations which motivated the problem of the classical absolute stability.

Following the definition of absolute stability initiated by Lur'e, we hereby introduce the notions of generalized sector and absolute contractive invariance as follows.

Definition 1: Given functions $\psi_{1}(u)$ and $\psi_{2}(u)$, each of which is concave or convex. A function $\psi(u, t)$, piecewise continuous in $t$ and locally Lipschitz in $u$, is said to satisfy a generalized sector condition if

$$
\psi(u, t) \in \operatorname{co}\left\{\psi_{1}(u), \psi_{2}(u)\right\} \quad \forall u, t \in \mathbf{R} .
$$

We use $\operatorname{co}\left\{\psi_{1}, \psi_{2}\right\}$ to denote the generalized sector, i.e., the set of functions that satisfy the above generalized sector condition. A level set $L_{V}(\rho)$ is said to be absolutely contractively invariant (ACI) over the sector $\operatorname{co}\left\{\psi_{1}, \psi_{2}\right\}$ if it is contractively invariant for (2) under every possible $\psi(u, t)$ satisfying the generalized sector condition. 
We see that if $L_{V}(\rho)$ is ACI, then any trajectory starting from it will converge to the origin under all $\psi(u, t)$ satisfying the generalized sector condition. Hence, $L_{V}(\rho)$ is an absolute stability region. We may use more general functions to define the boundary of a sector to capture more detail about a nonlinear component. The reason that we have chosen concave/convex functions is for the simplicity and completeness of the results. As we will see, the concavity and convexity of the boundary functions ensure that all the conditions for ACI are necessary and sufficient. We may also choose asymmetric functions or even symmetric functions. We have settled on odd symmetric functions since we will be focusing on ellipsoidal level set $L_{V}(\rho)$, which is symmetric about the origin. Let us next state a simple fact.

Fact 2: Given a level set $L_{V}(\rho)$ and a set of functions $\psi_{i}(u)$, $i \in I[1, N]$. Suppose that for each $i \in I[1, N], L_{V}(\rho)$ is contractively invariant for

$$
\dot{x}=A x+B \psi_{i}(F x) .
$$

Let $\psi(u, t)$ be a function such that $\psi(u, t) \in \operatorname{co}\left\{\psi_{i}(u), i \in\right.$ $I[1, N]\}$ for all $u \in \mathbf{R}$ and $t \in \mathbf{R}$, then $L_{V}(\rho)$ is contractively invariant for

$$
\dot{x}=A x+B \psi(F x, t) .
$$

This fact follows directly from the definition of contractive invariance [see (3)] and the differentiability of $V(x)$. Here $\psi_{i}(u)$ and $\psi(u, t)$ can be any nonlinear functions.

By Fact 2, we see that the absolute contractive invariance of a level set is equivalent to its contractive invariance under both $\psi_{1}(u)$ and $\psi_{2}(u)$. In view of this, we will focus on the invariance of a level set under a concave or convex function.

\section{Condition for Set Invariance Under Saturation Nonlinearity}

In [14], we obtained a simple necessary and sufficient condition for the invariance of an ellipsoid under saturation nonlinearity. This condition will be the foundation for all the main results in this paper.

Proposition 1: An ellipsoid $\mathcal{E}(P, \rho)$ is contractively invariant for

$$
\dot{x}=A x+B \operatorname{sat}(F x)
$$

if and only if

$$
(A+B F)^{T} P+P(A+B F)<0
$$

and there exists an $H \in \mathbf{R}^{1 \times n}$ such that

$$
(A+B H)^{T} P+P(A+B H)<0
$$

and $\mathcal{E}(P, \rho) \subset \mathcal{L}(H)$.

\section{INVARIANT ElLIPSOIDS UNDER CONCAVE OR CONVEX FUNCTIONS}

\section{A. Piecewise Linear Functions With One Bend}

Consider the following system:

$$
\dot{x}=A x+B \psi(F x)
$$

where $\psi(\cdot)$ is odd symmetric and

$$
\begin{aligned}
\psi(u) & =\left\{\begin{array}{ll}
k_{0} u, & \text { if } u \in\left[0, b_{1}\right] \\
k_{1} u+c_{1}, & \text { if } u \in\left(b_{1}, \infty\right)
\end{array}, \quad k_{0}>0\right. \\
b_{1} & =\frac{c_{1}}{\left(k_{0}-k_{1}\right)}>0 .
\end{aligned}
$$

Theorem 1: An ellipsoid $\mathcal{E}(P, \rho)$ is contractively invariant for (7) if and only if

$$
\left(A+k_{0} B F\right)^{T} P+P\left(A+k_{0} B F\right)<0
$$

and there exists an $H \in \mathbf{R}^{1 \times n}$ such that

$$
(A+B H)^{T} P+P(A+B H)<0
$$

and

$$
\mathcal{E}(P, \rho) \subset \mathcal{L}\left(\frac{H-k_{1} F}{c_{1}}\right) .
$$

Proof: We will prove the theorem by converting (7) into (4) and invoking Proposition 1. It can be verified that

$$
\psi(u)=k_{1} u+\left(k_{0}-k_{1}\right) b_{1} \operatorname{sat}\left(\frac{u}{b_{1}}\right)
$$

by noticing that $c_{1}=\left(k_{0}-k_{1}\right) b_{1}$. Therefore, (7) can be written as

$$
\dot{x}=\left(A+k_{1} B F\right) x+B\left(k_{0}-k_{1}\right) b_{1} \operatorname{sat}\left(\frac{F x}{b_{1}}\right) .
$$

Define $A_{\text {new }}:=A+k_{1} B F, B_{\text {new }}:=B\left(k_{0}-k_{1}\right) b_{1}$ and $F_{\text {new }}:=F / b_{1}$. Then, (7) is equivalent to

$$
\dot{x}=A_{\text {new }} x+B_{\text {new }} \operatorname{sat}\left(F_{\text {new }} x\right) .
$$

From Proposition 1, the necessary and sufficient condition for $\mathcal{E}(P, \rho)$ to be contractively invariant is

$$
\left(A_{\text {new }}+B_{\text {new }} F_{\text {new }}\right)^{T} P+P\left(A_{\text {new }}+B_{\text {new }} F_{\text {new }}\right)<0
$$

and there exists an $H_{\text {new }} \in \mathbf{R}^{1 \times n}$ such that

$$
\left(A_{\text {new }}+B_{\text {new }} H_{\text {new }}\right)^{T} P+P\left(A_{\text {new }}+B_{\text {new }} H_{\text {new }}\right)<0
$$

and $\mathcal{E}(P, \rho) \subset \mathcal{L}\left(H_{\text {new }}\right)$.

It can be verified that

$$
\begin{aligned}
A_{\text {new }}+B_{\text {new }} F_{\text {new }} & =A+k_{1} B F+B\left(k_{0}-k_{1}\right) b_{1}\left(\frac{F}{b_{1}}\right) \\
& =A+k_{0} B F
\end{aligned}
$$

and

$$
\begin{aligned}
A_{\text {new }}+B_{\text {new }} H_{\text {new }} & =A+k_{1} B F+B\left(k_{0}-k_{1}\right) b_{1} H_{\text {new }} \\
& =A+B\left(k_{1} F+\left(k_{0}-k_{1}\right) b_{1} H_{\text {new }}\right) .
\end{aligned}
$$

If we define $H=k_{1} F+\left(k_{0}-k_{1}\right) b_{1} H_{\text {new }}$, then (12) is equivalent to (8), (13) is equivalent to (9), and $\mathcal{E}(P, \rho) \subset \mathcal{L}\left(H_{\text {new }}\right)$ is equivalent to (10). The proof is completed by observing that $H_{\text {new }}$ is also uniquely determined by $H$.

The foregoing proof invoked Proposition 1 whose proof is quite involved. Here, we would like to provide some physical 
interpretation for the sufficiency of the condition. We need to show that

$$
x^{T} P(A x+B \psi(F x))<0 \quad \forall x \in \mathcal{E}(P, \rho) \backslash\{0\} .
$$

Assume that $\psi(u)$ is a concave function (the explanation for the case where $\psi(u)$ is convex is similar). For a concave function $\psi(u)$, we have $k_{1}<k_{0}, c_{1}>0$ and the set inclusion condition (10) can be equivalently written as

$$
k_{1} F x-c_{1} \leq H x \leq k_{1} F x+c_{1} \quad \forall x \in \mathcal{E}(P, \rho) .
$$

Notice that

$$
\psi(F x)=k_{0} F x, \quad \text { if }|F x| \leq b_{1} .
$$

Since $\psi(u)$ is concave for $u>0$, we have

$$
\psi(F x)=k_{1} F x+c_{1} \leq k_{0} F x, \quad \text { if } F x>b_{1}
$$

and

$$
\psi(F x)=k_{1} F x-c_{1} \geq k_{0} F x, \quad \text { if } F x<-b_{1} .
$$

It follows from (15) that for all $x \in \mathcal{E}(P, \rho)$,

$$
H x \leq \psi(F x) \leq k_{0} F x, \quad \text { if } F x>b_{1}
$$

and

$$
k_{0} F x \leq \psi(F x) \leq H x, \quad \text { if } F x<-b_{1} .
$$

In any case, we have

$$
\psi(F x) \in \operatorname{co}\left\{k_{0} F x, H x\right\} \quad \forall x \in \mathcal{E}(P, \rho) .
$$

Therefore

$$
\begin{aligned}
& x^{T} P(A x+B \psi(F x)) \\
& \leq \max \left\{x^{T} P\left(A+k_{0} B F\right) x, x^{T} P(A+B H) x\right\} .
\end{aligned}
$$

In view of (8) and (9), we obtain (14). The crucial point in the sufficiency is (16), which says that given the set inclusion condition (10) or (15), the nonlinear function $\psi(F x)$ is in the convex hull of two linear functions $H x$ and $k_{0} F x$.

With a given $P>0$, we would like to find the largest contractively invariant ellipsoid. Denote the largest $\rho$ (or the supreme of $\rho$ ) such that $\mathcal{E}(P, \rho)$ is contractively invariant for (7) as $\rho^{*}$. Suppose that (8) is satisfied (if not so, $\rho^{*}=0$ ). By Theorem 1

$$
\begin{aligned}
& \rho^{*}=\sup _{H} \rho \\
& \text { s.t. } \quad \text { a) } \mathcal{E}(P, \rho) \subset \mathcal{L}\left(\frac{H-k_{1} F}{c_{1}}\right) \\
& \quad \text { b) }(A+B H)^{T} P+P(A+B H)<0 .
\end{aligned}
$$

This optimization problem can be easily solved by using the LMI technique in [4]. Note that constraint a) is equivalent to (see [13])

$$
\left[\begin{array}{cc}
\frac{1}{\rho} & \frac{H-k_{1} F}{c_{1}} \\
\left(\frac{H-k_{1} F}{c_{1}}\right)^{T} & P
\end{array}\right] \geq 0 .
$$

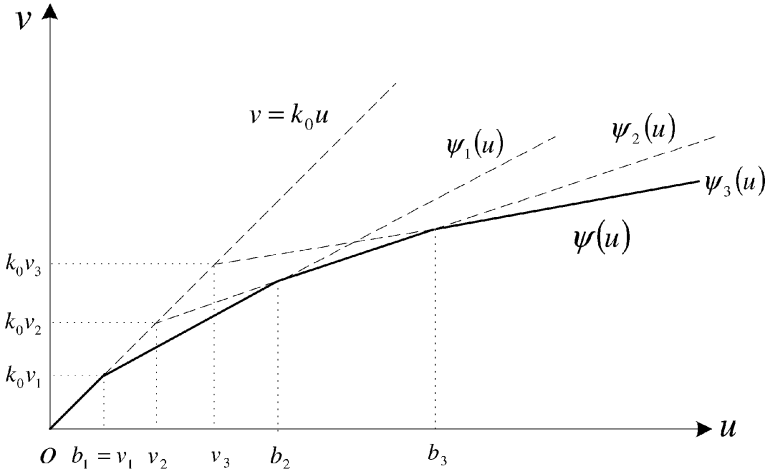

Fig. 7. Illustration for the proof of Theorem 2.

\section{B. Piecewise Linear Functions With Multiple Bends}

Consider the following system:

$$
\dot{x}=A x+B \psi(F x)
$$

where $\psi(\cdot)$ is continuous, odd symmetric, and

$$
\psi(u)= \begin{cases}k_{0} u, & \text { if } u \in\left[0, b_{1}\right] \\ k_{1} u+c_{1}, & \text { if } u \in\left(b_{1}, b_{2}\right] \\ \vdots & \\ k_{N} u+c_{N}, & \text { if } u \in\left(b_{N}, \infty\right) .\end{cases}
$$

The necessary and sufficient condition for an ellipsoid to be contractively invariant can also be obtained.

Theorem 2: Assume that $\psi(\cdot)$ is concave (or convex). An ellipsoid $\mathcal{E}(P, \rho)$ is contractively invariant for (18) if and only if

$$
\left(A+k_{0} B F\right)^{T} P+P\left(A+k_{0} B F\right)<0
$$

and there exist $H_{i} \in \mathbf{R}^{1 \times n}, i \in I[1, N]$, such that

$$
\left(A+B H_{i}\right)^{T} P+P\left(A+B H_{i}\right)<0 \quad i \in I[1, N]
$$

and

$$
\mathcal{E}(P, \rho) \subset \bigcap_{i=1}^{N} \mathcal{L}\left(\frac{H_{i}-k_{i} F}{c_{i}}\right) .
$$

Proof: The main idea of the proof is to construct $N$ piecewise linear functions $\psi_{i}(u)$, each of which has only one bend, such that for all $u \in \mathbf{R}$

$$
\psi(u) \in \operatorname{co}\left\{\psi_{1}(u), \psi_{2}(u), \ldots, \psi_{N}(u)\right\}
$$

and, meanwhile

$$
\psi_{i}(u) \in \operatorname{co}\left\{\psi(u), k_{0} u\right\} \quad i \in I[1, N] .
$$

This is possible due to the concavity (or convexity) of $\psi(u)$.

We only give the proof for the case where $\psi(u)$ is concave. The case where $\psi(u)$ is convex is similar. Let $v_{i}=c_{i} /\left(k_{0}-k_{i}\right)$. Then, $\left(v_{i}, k_{0} v_{i}\right)$ is the intersection of the straight line $v=k_{0} u$ with the other straight line obtained by extending the $(i+1)$ th section of $\psi(u)$ (see Fig. 7).

Define odd symmetric functions

$$
\psi_{i}(u):= \begin{cases}k_{0} u, & \text { if } u \in\left[0, v_{i}\right] \\ k_{i} u+c_{i}, & \text { if } u \in\left(v_{i}, \infty\right) .\end{cases}
$$


Then

$$
\psi(u)=\left\{\begin{array}{ll}
\psi_{1}(u), & \text { if } u \in\left[0, b_{2}\right] \\
\psi_{i}(u), & \text { if } u \in\left(b_{i}, b_{i+1}\right], \\
\psi_{N}(u), & \text { if } u \in\left(b_{N}, \infty\right)
\end{array} \quad i=2, \ldots, N-1\right.
$$

It follows that

$$
\psi(u) \in \operatorname{co}\left\{\psi_{1}(u), \psi_{2}(u), \ldots, \psi_{N}(u)\right\} \quad \forall u \in \mathbf{R} .
$$

It is also easy to see that for every $i \in I[1, N]$

$$
\psi(u) \leq \psi_{i}(u) \leq k_{0} u \quad \forall u>0
$$

due to the concavity of $\psi(u)$ (refer to Fact 1 ). By odd symmetry

$$
k_{0} u \leq \psi_{i}(u) \leq \psi(u) \quad \forall u<0
$$

In summary, for every $i \in I[1, N]$

$$
\psi_{i}(u) \in \operatorname{co}\left\{\psi(u), k_{0} u\right\} \quad \forall u \in \mathbf{R} .
$$

It is easy to see that (20) is a necessary condition for the contractive invariance of an ellipsoid $\mathcal{E}(P, \rho)$. Hence, we assume that it is true. From Fact 2 and by (24), the invariance of the ellipsoid $\mathcal{E}(P, \rho)$ for (18) implies its invariance for

$$
\dot{x}=A x+B \psi_{i}(F x), \quad i \in I[1, N] .
$$

On the other hand, by $(23)$, the invariance of $\mathcal{E}(P, \rho)$ for all the systems in (25) ensures its invariance for (18). Therefore, the invariance of $\mathcal{E}(P, \rho)$ for (18) is equivalent to its invariance for all the systems in (25). By Theorem 1, the latter is equivalent to the condition of Theorem 2 .

For a fixed $P$, the largest contractively invariant ellipsoid can also be obtained by solving an LMI optimization problem. Denote the largest $\rho$ such that $\mathcal{E}(P, \rho)$ is contractively invariant for (18) as $\rho^{*}$. Suppose that (20) is satisfied. By Theorem 2

$$
\begin{aligned}
& \rho^{*}=\sup _{H_{1}, \ldots, H_{N}} \rho \\
& \text { s.t. a) } \mathcal{E}(P, \rho) \subset \mathcal{L}\left(\frac{H_{i}-k_{i} F}{c_{i}}\right), \quad i \in I[1, N] \\
& \text { b) }\left(A+B H_{i}\right)^{T} P+P\left(A+B H_{i}\right)<0 \\
& \quad i \in I[1, N] .
\end{aligned}
$$

\section{Differentiable Functions}

Consider the following system:

$$
\dot{x}=A x+B \psi(F x)
$$

where $\psi(\cdot)$ is a continuously differentiable concave (or convex) function. Numerically, this $\psi(\cdot)$ can be approximated with piecewise linear concave functions by increasing the number of bending points and decreasing the distance between the neighboring bending points. Hence, a set invariance condition can be generalized from Theorem 2 by intuition. Here, we would like to provide an exact characterization of invariant ellipsoids.

Denote $k(z)=\left.(d \psi / d u)\right|_{u=z}$ and let $k_{0}=k(0)$. For $z>0$, draw a straight line that is tangential to $\psi(u)$ at $(z, \psi(z)$. Let the

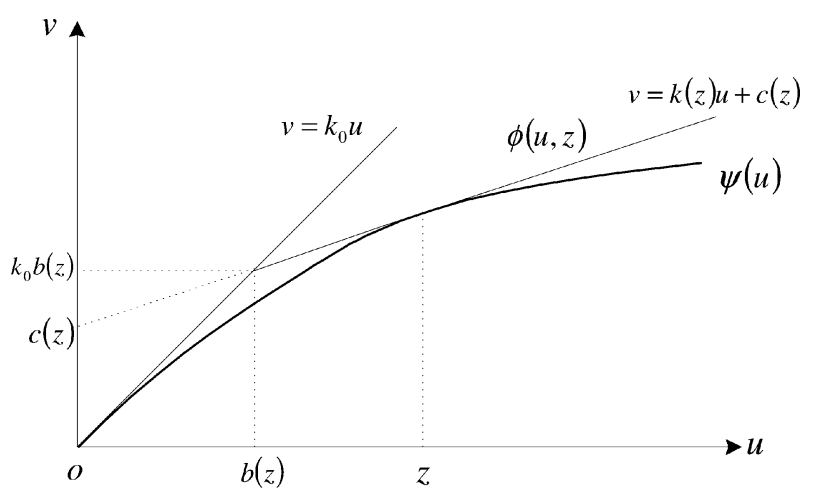

Fig. 8. Illustration for the definition of $\phi(u, z)$.

intersection of the straight line with $v=k_{0} u$ be $\left(b(z), k_{0} b(z)\right)$ and let the intersection of the straight line with the vertical axis be $(0, c(z))$ (see Fig. 8). Then

$$
\begin{aligned}
& c(z)=\psi(z)-k(z) z \\
& b(z)=\frac{c(z)}{k_{0}-k(z)}=\frac{\psi(z)-k(z) z}{k_{0}-k(z)} .
\end{aligned}
$$

Since $\psi(z)$ is concave, we have $\psi(z) \leq k_{0} z$ for all $z>0$ and, hence, $b(z) \leq z$.

Define

$$
\phi(u, z)= \begin{cases}k_{0} u, & \text { if } u \in[0, b(z)] \\ k(z) u+c(z), & \text { if } u \in(b(z), \infty) .\end{cases}
$$

For a fixed $z$, the function $\phi(\cdot, z)$ is a concave (or convex) piecewise linear function with one bend at $\left(b(z), k_{0} b(z)\right)$. Since $z \geq$ $b(z)$, we have

$$
\phi(z, z)=k(z) z+c(z)=k(z) z+\psi(z)-k(z) z=\psi(z) .
$$

Theorem 3: An ellipsoid $\mathcal{E}(P, \rho)$ is contractively invariant for (27) if and only if

$$
\left(A+k_{0} B F\right)^{T} P+P\left(A+k_{0} B F\right)<0
$$

and for every $z>0$, there exists an $H(z) \in \mathbf{R}^{1 \times n}$ such that

$$
(A+B H(z))^{T} P+P(A+B H(z))<0
$$

and

$$
\mathcal{E}(P, \rho) \subset \bigcap_{z>0} \mathcal{L}\left(\frac{H(z)-k(z) F}{c(z)}\right) .
$$

Proof: The idea is quite similar to the proof of Theorem 2. Since $\psi(u)=\phi(u, u)$, we have

$$
\psi(u) \in \operatorname{co}\{\phi(u, z): z>0\} \quad \forall u \in \mathbf{R} .
$$

On the other hand, by the concavity (or the convexity) of $\psi(u)$, we have, for every $z>0$,

$$
\phi(u, z) \in \operatorname{co}\left\{\psi(u), k_{0} u\right\} \quad \forall u \in \mathbf{R} .
$$

Hence, the invariance of $\mathcal{E}(P, \rho)$ for (27) is equivalent to its invariance for

$$
\dot{x}=A x+B \phi(F x, z)
$$




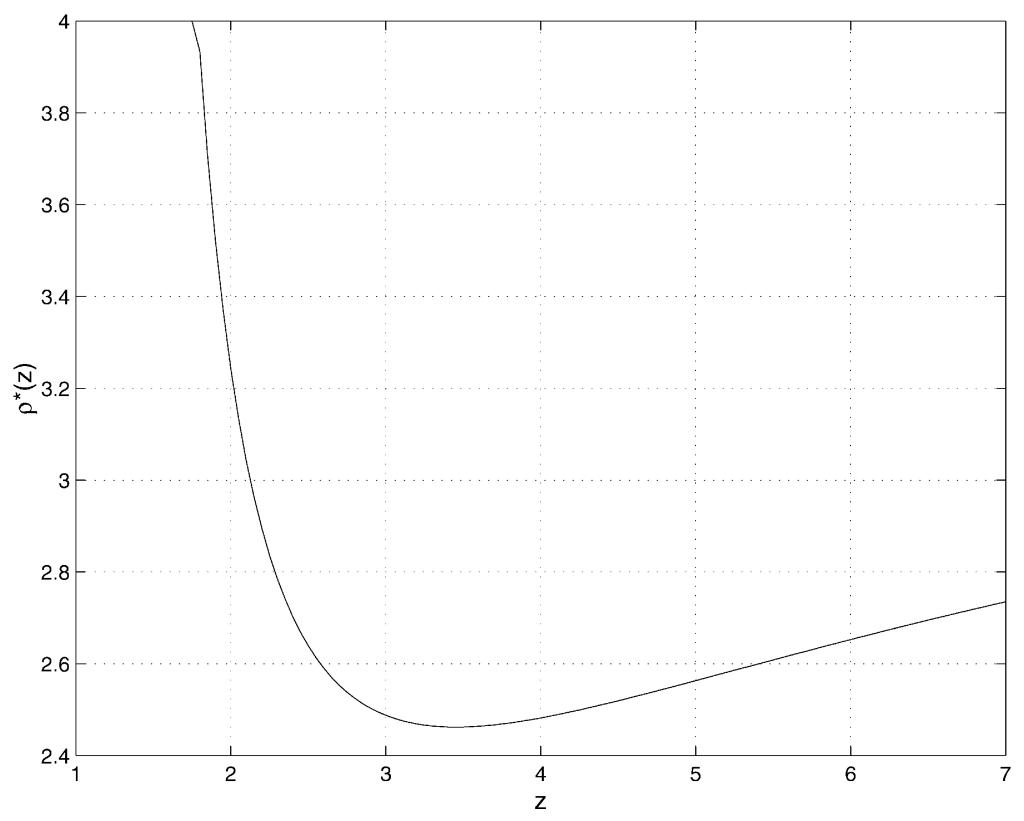

Fig. 9. Function $\rho^{*}(z)$.

for all $z>0$, which is in turn equivalent to the condition of this theorem by Theorem 1 .

Let $P$ be fixed. Denote the largest $\rho$ such that $\mathcal{E}(P, \rho)$ is contractively invariant for (27) as $\rho_{\max }$. Suppose that (28) is satisfied. Denote the largest $\rho$ such that $\mathcal{E}(P, \rho)$ is contractively invariant for $\dot{x}=A x+B \phi(F x, z)$ as $\rho^{*}(z)$, then

$$
\begin{aligned}
& \rho^{*}(z)=\sup _{H} \rho \\
& \text { s.t. } \quad \text { a) } \mathcal{E}(P, \rho) \subset \mathcal{L}\left(\frac{H-k(z) F}{c(z)}\right) \\
& \quad \text { b) }(A+B H)^{T} P+P(A+B H)<0
\end{aligned}
$$

and $\rho_{\max }=\inf \left\{\rho^{*}(z): z>0\right\}$.

Example 1: Consider system (27) with

$$
A=\left[\begin{array}{cc}
0.6 & -0.8 \\
0.8 & 0.6
\end{array}\right] \quad B=\left[\begin{array}{l}
2 \\
4
\end{array}\right]
$$

and $\psi(u)=\tan ^{-1}(u)$. The matrix $F$ is a feedback matrix which is designed by LQR method with $Q=I$ and $R=1$. The resulting $F$ and $P$ are

$$
F=\left[\begin{array}{ll}
1.2231 & -2.2486
\end{array}\right] \quad P=\left[\begin{array}{cc}
2.4628 & -1.5372 \\
-1.5372 & 1.3307
\end{array}\right] \text {. }
$$

It turns out that $\rho^{*}(z)=\infty$ for $z$ in the interval $\left[0, z_{0}\right]$, where $z_{0}=1.1662$ is the maximal $z$ such that

$$
(A+k(z) B F)^{T} P+P(A+k(z) B F) \leq 0 .
$$

For this example, $\rho^{*}(z)$ is a convex function for $z>z_{0}$ (see Fig. 9, where the function is clipped from above $\rho^{*}(z)=4$ so that its shape is clearly seen). The minimum value is reached at $z=3.45$ with $\rho^{*}(3.45)=2.4622$. Hence, the maximal invariant ellipsoid is $\mathcal{E}\left(P, \rho_{\max }\right)=\mathcal{E}(P, 2.4622)$. To verify that $\mathcal{E}\left(P, \rho_{\max }\right)$ is actually the largest invariant ellipsoid, we plotted the value of $\dot{V}(x)$ along the boundary of $\mathcal{E}\left(P, \rho_{\max }\right)$ in Fig. 10 (the solid curve), where the abscissa is the angle of $x \in \partial \mathcal{E}\left(P, \rho_{\max }\right)$. We also plotted $\dot{V}(x)$ along the boundaries of $\mathcal{E}\left(P, \rho_{\max }+0.3\right)$ (in dash-dotted curve) and $\mathcal{E}\left(P, \rho_{\max }-0.3\right)$ (in dashed curve) in Fig. 10 as comparison. We see clearly that $\dot{V}(x) \leq 0$ for all $x \in \partial \mathcal{E}\left(P, \rho_{\max }\right)$ and there are two points on $\partial \mathcal{E}\left(P, \rho_{\max }\right)$ where $\dot{V}(x)=0$. As $\rho$ is increased, $\dot{V}(x)>0$ for some segment and as $\rho$ is decreased, $\dot{V}(x)<0$ for all $x \in \partial \mathcal{E}(P, \rho)$.

We also determined the largest invariant ellipsoid of the given shape that can be confirmed by the vertex criterion in [18] (the vertex criterion is better than the circle criterion as was implied in [18]). To do this, we first calculated the minimal $k$ such that

$$
(A+k B F)^{T} P+P(A+k B F) \leq 0
$$

which is $k=0.4237$. Then we located the point on $\tan ^{-1}(u)$ such that $\tan ^{-1}(u) / u=0.4237$, which is $u=2.9310$. The largest invariant $\mathcal{E}(P, \rho)$ that can be concluded from vertex criterion is the one such that $\mathcal{E}(P, \rho)$ lies between the two straight lines $F x= \pm 2.9310$, i.e., $\mathcal{E}(P, \rho) \subset \mathcal{L}(F / 2.9310)$, which is $\mathcal{E}(P, 1.3290)$. We see that the largest invariant $\mathcal{E}(P, \rho)$ that can be concluded from vertex criterion is much smaller than the one we obtained using Theorem 3, which is the actual largest invariant ellipsoid of the given shape. Fig. 11 plots both $\mathcal{E}\left(P, \rho_{\max }\right)$ and $\mathcal{E}(P, 1.3290)$ for comparison.

\section{CONVEX Hull of INVARIANT ElLIPSOIDS}

In this section, we will show that under a concave or convex nonlinearity, the convex hull of a set of invariant ellipsoids is also invariant. This result will lead to potentially much larger estimation of the domain of attraction. To establish this result, we need to resort to the composite quadratic Lyapunov function that we introduced in [15]. 


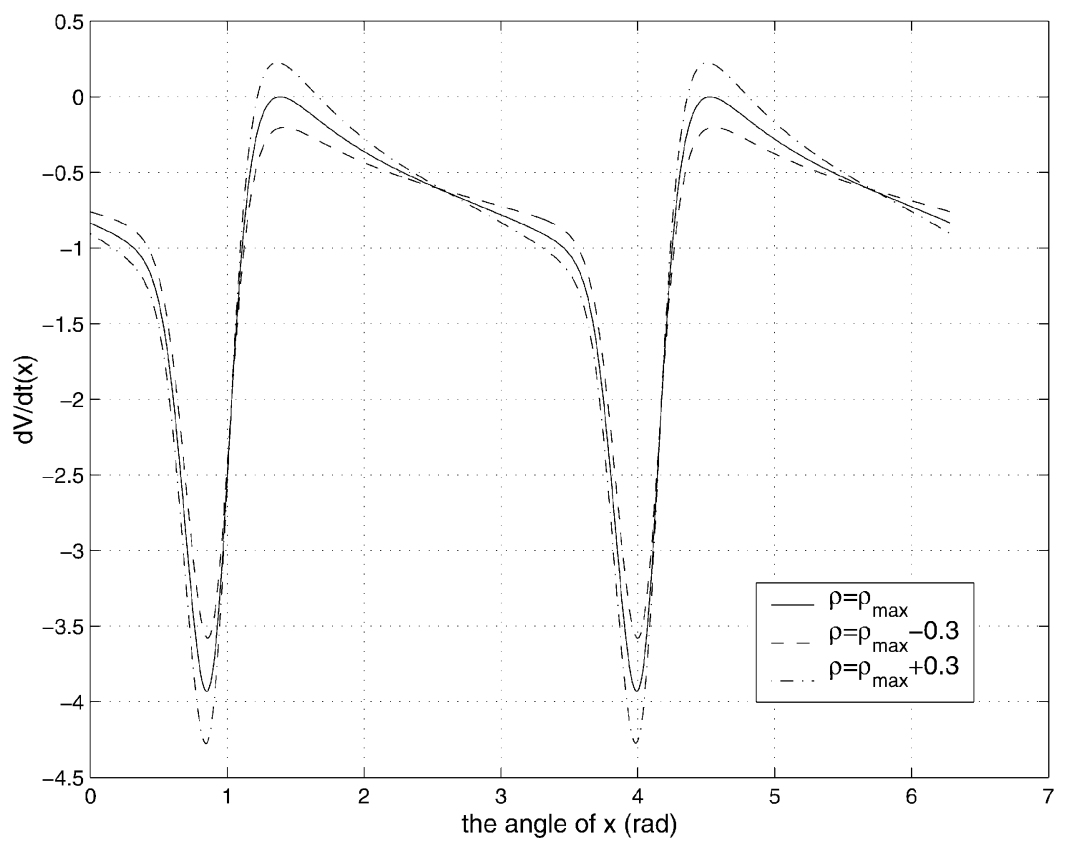

Fig. 10. Derivative of $V(x)$ along the boundaries of ellipsoids.

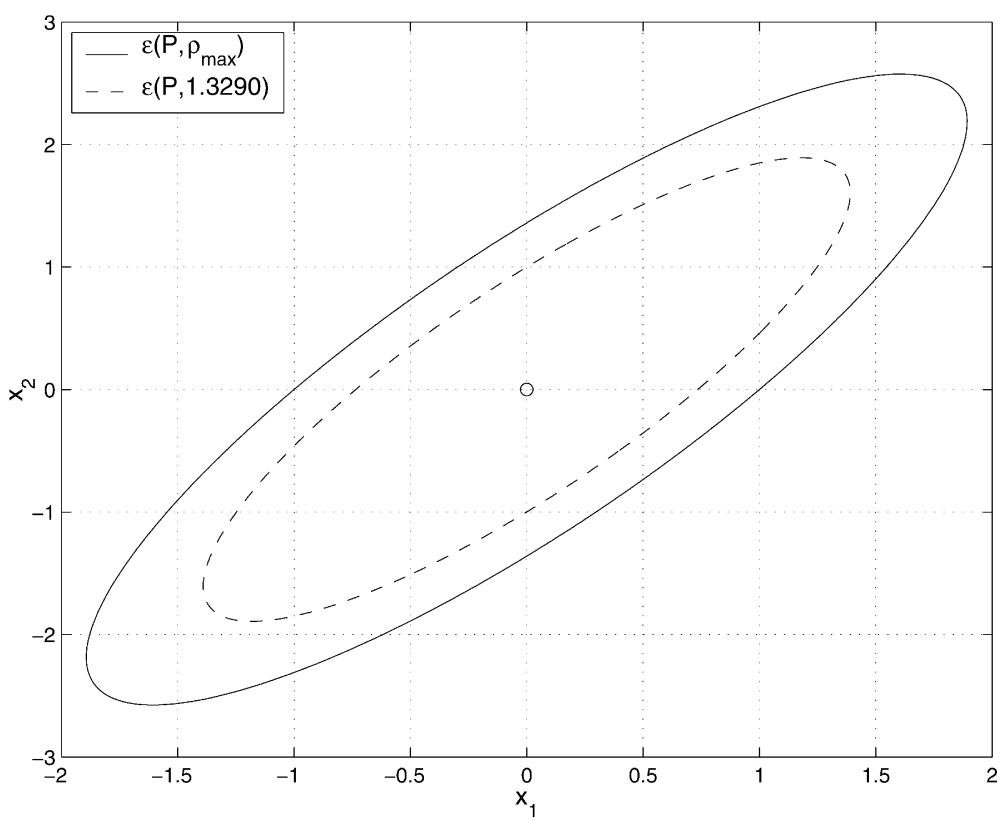

Fig. 11. Comparison of the largest invariant ellipsoids by different methods.

\section{A. The Composite Quadratic Lyapunov Function}

The composite quadratic Lyapunov function is determined by a set of positive-definite matrices $P_{1}, P_{2}, \ldots, P_{J} \in \mathbf{R}^{n \times n}$. Let $Q_{j}=P_{j}^{-1}, j \in I[1, J]$. For a vector $\gamma \in \mathbf{R}^{J}$, define

$$
Q(\gamma):=\sum_{j=1}^{J} \gamma_{j} Q_{j} \quad P(\gamma):=Q^{-1}(\gamma)
$$

Let

$$
\Gamma=\left\{\gamma \in \mathbf{R}^{J}: \gamma_{1}+\gamma_{2}+\cdots+\gamma_{J}=1, \gamma_{j} \geq 0\right\}
$$

It is easy to see that $Q(\gamma), P(\gamma)>0$ for all $\gamma \in \Gamma$ and these two matrix functions are analytic in $\gamma \in \Gamma$. The composite quadratic function is defined as

$$
V_{c}(x):=\min _{\gamma \in \Gamma} x^{T} P(\gamma) x .
$$

For $\rho>0$, the level set of $V_{c}(x)$ is

$$
L_{V_{c}}(\rho):=\left\{x \in \mathbf{R}^{n}: V_{c}(x) \leq \rho\right\} .
$$

Denote the convex hull of the ellipsoids $\mathcal{E}\left(P_{j}, \rho\right), j \in I[1, J]$, as

$\operatorname{co}\left\{\mathcal{E}\left(P_{j}, \rho\right), j \in I[1, J]\right\}$

$$
:=\left\{\sum_{j=1}^{J} \gamma_{j} x_{j}: x_{j} \in \mathcal{E}\left(P_{j}, \rho\right), \gamma \in \Gamma\right\} .
$$


The following properties about this composite quadratic function were established in [15].

Theorem 4:

1) $L_{V_{c}}(\rho)=\operatorname{co}\left\{\mathcal{E}\left(P_{j}, \rho\right), j \in I[1, J]\right\}=$ $\bigcup_{\gamma \in \Gamma} \mathcal{E}(P(\gamma), \rho)$

2) The function $V_{c}(x)$ is continuously differentiable. Let $\gamma^{*}$ be an optimal $\gamma$ such that $x^{T} P\left(\gamma^{*}\right) x=$ $\min _{\gamma \in \Gamma} x^{T} P(\gamma) x$, then

$$
\frac{\partial V_{c}}{\partial x}=2 P\left(\gamma^{*}\right) x
$$

The function $V_{c}(x)$ and an optimal $\gamma$ can be computed with the LMI technique in [4]

$$
\begin{aligned}
& V_{c}(x)=\min _{\gamma} \alpha \\
& \text { s.t. }\left[\begin{array}{ll}
\alpha & x^{T} \\
x & \sum_{j=1}^{J} \gamma_{j} Q_{j}
\end{array}\right] \geq 0 \\
& \sum_{j=1}^{J} \gamma_{j}=1, \quad \gamma_{j} \geq 0 .
\end{aligned}
$$

The following fact will also be useful for establishing the main results of this section.

Fact 3 [13]: For a vector $f_{0} \in \mathbf{R}^{1 \times n}$ and a matrix $P>0$, $\mathcal{E}(P) \subset \mathcal{L}\left(f_{0}\right)$ if and only if

$$
f_{0} P^{-1} f_{0}^{T} \leq 1 \Longleftrightarrow\left[\begin{array}{cc}
1 & f_{0} P^{-1} \\
P^{-1} f_{0}^{T} & P^{-1}
\end{array}\right] \geq 0 .
$$

1) The equality $f_{0} P^{-1} f_{0}^{T}=1$ holds if and only if the ellipsoid $\mathcal{E}(P)$ touches the hyperplane $f_{0} x=1$ at $x_{0}=$ $P^{-1} f_{0}^{T}$ (the only intersection), i.e.,

$$
1=f_{0} x_{0}>f_{0} x \quad \forall x \in \mathcal{E}(P) \backslash\left\{x_{0}\right\} .
$$

2) If $f_{0} P^{-1} f_{0}^{T}<1$, then

$$
\left[\begin{array}{cc}
1 & f_{0} P^{-1} \\
P^{-1} f_{0}^{T} & P^{-1}
\end{array}\right]>0
$$

and the ellipsoid $\mathcal{E}(P)$ lies strictly between the hyperplanes $f_{0} x=1$ and $f_{0} x=-1$ without touching them.

\section{B. Invariance of the Convex Hull of Ellipsoids}

Theorem 5: Let $\psi(\cdot): \mathbf{R} \mapsto \mathbf{R}$ be a concave function. Consider the system

$$
\dot{x}=A x+B \psi(F x) \text {. }
$$

Given a set of ellipsoids $\mathcal{E}\left(P_{j}\right), j \in I[1, J]$. If each ellipsoid $\mathcal{E}\left(P_{j}\right)$ is contractively invariant for $(34)$, then $\operatorname{co}\left\{\mathcal{E}\left(P_{j}\right), j \in\right.$ $I[1, J]\}$ is a contractively invariant set.

Proof: For simplicity, we assume that $k_{0}=$ $\left.(d \psi / d u)\right|_{u=0}=1$. Otherwise, we can replace $B$ with $B k_{0}$ and $\psi(\cdot)$ with $\psi(\cdot) / k_{0}$. Since each ellipsoid $\mathcal{E}\left(P_{j}\right)$ is contractively invariant for (34), by Theorems 2 and 3, we have

$$
(A+B F)^{T} P_{j}+P_{j}(A+B F)<0, \quad j \in I[1, J] .
$$

Recall from Theorem 4 that $\operatorname{co}\left\{\mathcal{E}\left(P_{j}\right), j \in I[1, J]\right\}=$ $L_{V_{c}}(1)$. It suffices to show that

$\dot{V}_{c}(x)=\left(\frac{\partial V_{c}}{\partial x}\right)^{T}(A x+B \psi(F x))<0 \quad \forall x \in \partial L_{V_{c}}(1)$.

Denote $V_{j}(x)=x^{T} P_{j} x$. Now, we consider an arbitrary $x_{0} \in$ $\partial L_{V_{c}}(1)$. For simplicity, assume that $F x_{0} \geq 0$ (the proof for $F x_{0} \leq 0$ is similar). If $x_{0} \in \partial \mathcal{E}\left(P_{j}\right)$ for some $j \in I[1, J]$, then $\left.\left(\bar{\partial} V_{c} / \partial x\right)\right|_{x=x_{0}}=2 P_{j} x_{0}$ and $\dot{V}_{c}\left(x_{0}\right)=\dot{V}_{j}\left(x_{0}\right)<0$ follows from the invariance of $\mathcal{E}\left(P_{j}\right)$. Hence, we assume that $x_{0} \notin$ $\partial \mathcal{E}\left(P_{j}\right)$ for any $j$. Then, there exist an integer $J_{0} \leq J$, some numbers $\alpha_{j} \in(0,1)$ and vectors $x_{j} \in \mathcal{E}\left(P_{j}\right), j \in I\left[1, J_{0}\right]$, such that

$$
\sum_{j=1}^{J_{0}} \alpha_{j}=1 \quad x_{0}=\sum_{j=1}^{J_{0}} \alpha_{j} x_{j}
$$

(Here, we have assumed for simplicity that $x_{0}$ is only related to the first $J_{0}$ ellipsoids. Otherwise, the ellipsoids can be reordered to meet this assumption.) Let $h_{0}=(1 / 2)\left(\left.\left(\partial V_{c} / \partial x\right)\right|_{x=x_{0}}\right)^{T}$, then by Theorem $4, h_{0} x_{0}=x_{0}^{T} P\left(\gamma^{*}\right) x_{0}=1$. It follows that the hyperplane $h_{0} x=1$ is tangential to the convex set $L_{V_{c}}(1)$ at $x=x_{0}$. Hence, $L_{V_{c}}(1)$ lies between $h_{0} x=1$ and $h_{0} x=-1$, i.e., $L_{V_{c}}(1) \subset \mathcal{L}\left(h_{0}\right)$. Therefore

$$
\mathcal{E}\left(P_{j}\right) \subset \mathcal{L}\left(h_{0}\right) \quad \forall j \in I\left[1, J_{0}\right]
$$

and

$$
1=h_{0} x_{0} \geq h_{0} x_{j} \quad \forall j \in I\left[1, J_{0}\right] .
$$

We claim that $h_{0} x_{j}=1$ for all $j \in I\left[1, J_{0}\right]$. Suppose on the contrary that $h_{0} x_{j}<1$ for some $j$, say, $h_{0} x_{1}<1$, then

$$
\begin{aligned}
1 & =h_{0} x_{0}=\alpha_{1} h_{0} x_{1}+\sum_{j=2}^{J_{0}} \alpha_{j} h_{0} x_{j} \\
& \leq \alpha_{1} h_{0} x_{1}+\sum_{j=2}^{J_{0}} \alpha_{j}<\sum_{j=1}^{J_{0}} \alpha_{j}=1
\end{aligned}
$$

which is a contradiction. Because of (36) and $x_{j} \in \mathcal{E}\left(P_{j}\right)$, the equality $h_{0} x_{j}=1$ implies that $\mathcal{E}\left(P_{j}\right)$ touches the hyperplane $h_{0} x=1$ at $x=x_{j}$. Hence, the hyperplane $h_{0} x=1$ is tangential to $\mathcal{E}\left(P_{j}\right)$ at $x_{j}$ for every $j \in I\left[1, J_{0}\right]$. It follows from Fact 3 that

$$
h_{0}^{T}=P_{j} x_{j} \quad \forall j \in I\left[1, J_{0}\right] .
$$

Since each ellipsoid $\mathcal{E}\left(P_{j}\right)$ is contractively invariant, we have

$$
\begin{aligned}
\dot{V}_{j}\left(x_{j}\right) & =2 x_{j}^{T} P_{j}\left(A x_{j}+B \psi\left(F x_{j}\right)\right) \\
& =2 h_{0}\left(A x_{j}+B \psi\left(F x_{j}\right)\right)<0, \quad j \in I\left[1, J_{0}\right] .
\end{aligned}
$$

We need to show that

$$
\dot{V}_{c}\left(x_{0}\right)=2 h_{0}\left(A x_{0}+B \psi\left(F x_{0}\right)\right)<0 .
$$

Because of (35), we have

$$
\begin{gathered}
2 x_{j}^{T} P_{j}\left(A x_{j}+B F x_{j}\right)=2 h_{0}\left(A x_{j}+B F x_{j}\right)<0 \\
\forall j \in I\left[1, J_{0}\right] .
\end{gathered}
$$


Hence, for all $x \in \operatorname{co}\left\{x_{1}, x_{2}, \ldots, x_{J_{0}}\right\}$

$$
2 h_{0}(A x+B F x)<0 \text {. }
$$

We first assume that $F x_{j} \geq 0$ for all $j \in I\left[1, J_{0}\right]$. In this case, $F x \geq 0$ for all $x \in \operatorname{co}\left\{x_{1}, x_{2}, \ldots, x_{J_{0}}\right\}$. If $h_{0} B \geq 0$, then

$$
\dot{V}_{c}\left(x_{0}\right)=2 h_{0}\left(A x_{0}+B \psi\left(F x_{0}\right)\right) \leq 2 h_{0}\left(A x_{0}+B F x_{0}\right)<0
$$

noticing that, by the assumption that $F x_{0} \geq 0$ and by the property of the function $\psi(\cdot)$, we have $\psi\left(F x_{0}\right) \leq F x_{0}$. If $h_{0} B \leq 0$, then by the property of $\psi(\cdot), h_{0} A x+h_{0} B \psi(F x)$ is a convex function for $x \in \operatorname{co}\left\{x_{1}, x_{2}, \ldots, x_{J_{0}}\right\}$. Hence we also have (38) by (37).

If $F x_{j} \geq 0$ does not hold for all $j \in I\left[1, J_{0}\right]$, then we can get an intersection of the set $\operatorname{co}\left\{x_{1}, x_{2}, \ldots, x_{J_{0}}\right\}$ with the half space $F x \geq 0$. This intersection is also a polygon and can be denoted as $\operatorname{co}\left\{y_{1}, y_{2}, \ldots, y_{J_{1}}\right\}$. Since $F x_{0} \geq 0$, we have $x_{0} \in \operatorname{co}\left\{y_{1}, y_{2}, \ldots, y_{J_{1}}\right\}$. Some $y_{j}$ 's belong to $\left\{x_{1}, x_{2}, \ldots, x_{J_{0}}\right\}$, others are not. For those $y_{j} \notin\left\{x_{i}: i \in I\left[1, J_{0}\right]\right\}$, we must have $F y_{j}=0$ and $y_{j} \in \operatorname{co}\left\{x_{i}: i \in I\left[1, J_{0}\right]\right\}$. It follows from (39) that $h_{0}\left(A y_{j}+B F y_{j}\right) \leq 0$. Since $\psi(0)=0$, for those $y_{j}$ 's such that $F y_{j}=0$, we have

$$
\dot{V}_{c}\left(y_{j}\right)=2 h_{0}\left(A y_{j}+B \psi\left(F y_{j}\right)\right)=2 h_{0}\left(A y_{j}+B F y_{j}\right)<0 \text {. }
$$

In summary, we have

$$
\dot{V}_{c}\left(y_{j}\right)=2 h_{0}\left(A y_{j}+B \psi\left(F y_{j}\right)\right)<0 \quad \forall j \in I\left[1, J_{1}\right] .
$$

Because of this, we can work on the set $\operatorname{co}\left\{y_{1}, y_{2}, \ldots, y_{J_{1}}\right\}$ instead of $\operatorname{co}\left\{x_{1}, x_{2}, \ldots, x_{J_{0}}\right\}$. Since $F y_{j} \geq 0$ for all $j \in$ $I\left[1, J_{1}\right]$, same arguments can be used to prove (38) by using (40) instead of (37).

Actually, the result in Theorem 5 is also true if $\psi(u)$ is a convex function.

Theorem 6: Let $\psi(\cdot): \mathbf{R} \mapsto \mathbf{R}$ be a convex function such that $k_{\infty}:=\sup _{u>0}(\psi(u) / u)<\infty$. Consider the system

$$
\dot{x}=A x+B \psi(F x) .
$$

Given a set of ellipsoids $\mathcal{E}\left(P_{j}\right), j \in I[1, J]$. If each ellipsoid $\mathcal{E}\left(P_{j}\right)$ is contractively invariant for $(41)$, then $\operatorname{co}\left\{\mathcal{E}\left(P_{j}\right), j \in\right.$ $I[1, J]\}$ is a contractively invariant set.

Proof: Define $\phi(u)=k_{\infty} u-\psi(u)$. Then $\phi(\cdot)$ is a concave function and (41) can be written as

$$
\dot{x}=\left(A+k_{\infty} B F\right) x-B \phi(F x) .
$$

The result follows readily from Theorem 5 .

\section{Absolutely InVARIant SETS Under a GenERAlized SECTOR CONDITION}

Consider a system with a nonlinear component

$$
\dot{x}=A x+B \psi(F x, t)
$$

where $\psi(u, t)$ satisfies the generalized sector condition, i.e., $\psi(u, t) \in \operatorname{co}\left\{\psi_{1}(u), \psi_{2}(u)\right\}$ for all $u, t \in \mathbf{R}$, and each of $\psi_{1}(\cdot)$ and $\psi_{2}(\cdot)$ is a piecewise linear concave or convex function. Suppose that

$$
\psi_{1}(u)= \begin{cases}k_{01} u, & \text { if } u \in\left[0, b_{11}\right] \\ k_{11} u+c_{11}, & \text { if } u \in\left[b_{11}, b_{21}\right] \\ \vdots & \\ k_{N_{1} 1} u+c_{N_{1} 1}, & \text { if } u \in\left(b_{N_{1} 1}, \infty\right) .\end{cases}
$$

and

$$
\psi_{2}(u)= \begin{cases}k_{02} u, & \text { if } u \in\left[0, b_{12}\right] \\ k_{12} u+c_{12}, & \text { if } u \in\left[b_{12}, b_{22}\right] \\ \vdots & \\ k_{N_{2} 2} u+c_{N_{2} 2}, & \text { if } u \in\left(b_{N_{2} 2}, \infty\right) .\end{cases}
$$

As we have noted earlier, the absolute contractive invariance of an ellipsoid is equivalent to its invariance under both $\psi_{1}(u)$ and $\psi_{2}(u)$, which can be verified by Theorem 2 . Hence, we have the following.

Corollary 1: An ellipsoid $\mathcal{E}(P, \rho)$ is absolutely contractively invariant for (42) over the sector $\operatorname{co}\left\{\psi_{1}, \psi_{2}\right\}$ if and only if

$$
\begin{aligned}
& \left(A+k_{01} B F\right)^{T} P+P\left(A+k_{01} B F\right)<0 \\
& \left(A+k_{02} B F\right)^{T} P+P\left(A+k_{02} B F\right)<0
\end{aligned}
$$

and there exist $H_{i 1} \in \mathbf{R}^{1 \times n}, i \in I\left[1, N_{1}\right]$ and $H_{i 2} \in \mathbf{R}^{1 \times n}$, $i \in I\left[1, N_{2}\right]$ such that

$$
\begin{aligned}
& \left(A+B H_{i 1}\right)^{T} P+P\left(A+B H_{i 1}\right)<0 \\
& \left(A+B H_{i 2}\right)^{T} P+P\left(A+B H_{i 2}\right)<0
\end{aligned}
$$

and

$$
\begin{aligned}
& \mathcal{E}(P, \rho) \subset \bigcap_{i=1}^{N_{1}} \mathcal{L}\left(\frac{H_{i 1}-k_{i 1} F}{c_{i 1}}\right) \\
& \mathcal{E}(P, \rho) \subset \bigcap_{i=1}^{N_{2}} \mathcal{L}\left(\frac{H_{i 2}-k_{i 2} F}{c_{i 2}}\right) .
\end{aligned}
$$

An optimization problem can be formulated from Corollary 1 to find the largest $\rho$ such that $\mathcal{E}(P, \rho)$ is ACI. Here, all the conditions can be transformed into LMIs.

Now, suppose that we have a set of ACI ellipsoids. Then it is clear that each of these ellipsoid is contractively invariant under both $\psi_{1}(\cdot)$ and $\psi_{2}(\cdot)$. By Theorems 5 and 6 , the convex hull of these ellipsoids is also contractively invariant under both $\psi_{1}(\cdot)$ and $\psi_{2}(\cdot)$. By Fact 2 , we can be sure that the convex hull of these ellipsoids is ACI. In summary, we have the following.

Theorem 7: Consider the following system:

$$
\dot{x}=A x+B \psi(F x, t)
$$

where $\psi(u, t)$ satisfies a generalized sector bound $\operatorname{co}\left\{\psi_{1}, \psi_{2}\right\}$ and each of $\psi_{1}(u)$ and $\psi_{2}(u)$ is concave or convex. Suppose that we have a set of absolutely contractively invariant ellipsoids $\mathcal{E}\left(P_{j}, \rho_{j}\right), j \in I[1, J]$. Then the convex hull of these ellipsoids is also absolutely contractively invariant. 


\section{ESTIMATION OF THE DOMAIN OF ATTRACTION}

For a system with a generalized sector bound, there may exist infinitely many ellipsoids that are absolutely contractively invariant. By Theorem 7, the convex hull of all these ellipsoids form a larger absolutely contractively invariant set. Hence, this convex hull can be considered as an estimate of the domain of attraction. However, it is impossible to obtain all the absolutely contractively invariant ellipsoids. A practical approach is to choose a finite number of these ellipsoids such that each of them is the "largest one" according to some measure of set size.

Let $\mathcal{X}_{R} \subset \mathbf{R}^{n}$ be a bounded convex set of some desired shape. We call it a shape reference set. Suppose that $0 \in \mathcal{X}_{R}$. For a positive real number $\alpha$, denote

$$
\alpha \mathcal{X}_{R}=\left\{\alpha x: x \in \mathcal{X}_{R}\right\}
$$

For a set $\mathcal{S} \subset \mathbf{R}^{n}$, define the size of $\mathcal{S}$ with respect to $\mathcal{X}_{R}$ as

$$
\alpha_{R}(\mathcal{S}):=\sup \left\{\alpha>0: \alpha \mathcal{X}_{R} \subset \mathcal{S}\right\}
$$

Two typical types of $\mathcal{X}_{R}$ are the ellipsoids and the polyhedrons. A very special type of shape reference set is a straight line segment

$$
\mathcal{X}_{R}=\operatorname{co}\left\{x_{0},-x_{0}\right\}
$$

for some $x_{0} \in \mathbf{R}^{n}$. This type of $\mathcal{X}_{R}$ is very simple and yet very useful. By maximizing the invariant ellipsoid with respect to it, we obtain the point furthest to the origin along the direction of $x_{0}$ that can be enclosed by an invariant ellipsoid. Hence, we can conclude that this point is inside the domain of attraction. By taking different $x_{0}$, we will obtain different invariant ellipsoids, all maximized in certain sense (with respect to different $\mathcal{X}_{R}=$ $\operatorname{co}\left\{x_{0},-x_{0}\right\}$ ). By Theorem 7 , the convex hull of these ellipsoids gives us an estimate of the domain of attraction.

In the following, we present a method for maximizing the absolutely contractively invariant ellipsoid with respect to $\mathcal{X}_{R}=$ $\operatorname{co}\left\{x_{0},-x_{0}\right\}$ for a fixed $x_{0}$. By Corollary 1 , this problem can be formulated as follows:

$$
\begin{aligned}
& \sup _{P>0, H_{11}, \ldots, H_{N_{1} 1}, H_{12}, \ldots, H_{N_{2} 2}} \alpha \\
& \text { s.t. } \quad \text { a) } \mathcal{E}(P, \rho) \subset \mathcal{L}\left(\frac{H_{i 1}-k_{i 1} F}{c_{i 1}}\right), \quad i \in I\left[1, N_{1}\right] \\
& \text { b) } \mathcal{E}(P, \rho) \subset \mathcal{L}\left(\frac{H_{i 2}-k_{i 2} F}{c_{i 2}}\right), \quad i \in I\left[1, N_{2}\right] \\
& \text { c) }\left(A+B H_{i 1}\right)^{T} P+P\left(A+B H_{i 1}\right)<0 \\
& i \in I\left[1, N_{1}\right] \\
& \text { d) }\left(A+B H_{i 2}\right)^{T} P+P\left(A+B H_{i 2}\right)<0 \\
& i \in I\left[1, N_{2}\right] \\
& \text { e) }\left(A+k_{01} B F\right)^{T} P+P\left(A+k_{01} B F\right)<0 \\
& \text { f) }\left(A+k_{02} B F\right)^{T} P+P\left(A+k_{02} B F\right)<0 \\
& \text { g) } \alpha x_{0} \in \mathcal{E}(P, \rho) \text {. }
\end{aligned}
$$

This optimization problem can also be transformed into an LMI problem by defining new parameters. Let

$$
\gamma=\frac{1}{\alpha^{2}} \quad Q=\left(\frac{P}{\rho}\right)^{-1} \quad Y_{i 1}=H_{i 1} Q \quad Y_{i 2}=H_{i 2} Q
$$

Then it can be shown with the standard technique (see, e.g., [4] and [13]) that (49) is equivalent to

$$
\begin{aligned}
& Q>0, Y_{i 1}, \ldots, Y_{N_{11}}, Y_{12}, \ldots, Y_{N_{2} 2} \\
& \text { s.t. } \\
& \text { a) }\left[\begin{array}{cc}
1 & \frac{Y_{i 1}-k_{i 1} F Q}{c_{i 1}} \\
\frac{Y_{i 1}^{T}-k_{i 1} Q F^{T}}{c_{i 1}} & Q
\end{array}\right] \geq 0, \quad i \in I\left[1, N_{1}\right] \\
& \text { b) }\left[\begin{array}{cc}
1 & \frac{Y_{i 2}-k_{i 2} F Q}{c_{i 2}} \\
\frac{Y_{i 2}^{T}-k_{i 2} Q F^{T}}{c_{i 2}} & Q
\end{array}\right] \geq 0, \quad i \in I\left[1, N_{2}\right] \\
& \text { c) } Q A^{T}+A Q+Y_{i 1} B^{T}+B Y_{i 1}<0, \quad i \in I\left[1, N_{1}\right] \\
& \text { d) } Q A^{T}+A Q+Y_{i 2} B^{T}+B Y_{i 2}<0, \quad i \in I\left[1, N_{2}\right] \\
& \text { e) } Q\left(A+k_{01} B F\right)^{T}+\left(A+k_{01} B F\right) Q<0 \\
& \text { f) } Q\left(A+k_{02} B F\right)^{T}+\left(A+k_{02} B F\right) Q<0 \\
& \text { g) }\left[\begin{array}{cc}
\gamma & x_{0}^{T} \\
x_{0} & Q
\end{array}\right] \geq 0 .
\end{aligned}
$$

Example 2: Consider the same system as in Example 1. Although an optimization problem can be formulated from Theorem 3 to maximize the invariant ellipsoid with respect to a shape reference set, it will be an infinite dimensional problem. A feasible approach is to place $\tan ^{-1}(u)$ between two piecewise linear functions and then use the conditions for absolute contractive invariance. The first piecewise linear function can be simply taken as the linear function $\psi_{1}(u)=u$ (note that $k_{0}=1$ for $\tan ^{-1}(u)$ ) and the second piecewise linear function can be obtained by connecting a finite number of points on $\tan ^{-1}(u)$ including the origin. By the concavity of the function, $\tan ^{-1}(u) \in \operatorname{co}\left\{u, \psi_{2}(u)\right\}$ for all $u \in \mathbf{R}$.

Here, we select six points $\left(u, \tan ^{-1}(u)\right), u=0,1,2,3,5,8$. The resulting piecewise linear function $\psi_{2}(u)$ has the form of (19) with $N=5$

$$
\begin{aligned}
& \left(k_{0}, k_{1}, k_{2}, k_{3}, k_{4}, k_{5}\right) \\
& =(0.7845,0.3218,0.1419,0.0622,0.0243,0)
\end{aligned}
$$

and

$\left(c_{1}, c_{2}, c_{3}, c_{4}, c_{5}\right)=(0.4636,0.8234,1.0625,1.2517,1.4464)$.

Fig. 12 plots the functions $v=\tan ^{-1}(u)$ (in dashed curve), $v=\psi_{2}(u)$ (in solid curve) and the straight line $v=\psi_{1}(u)=u$. We choose $x_{0}=\left[\begin{array}{l}\cos (\theta) \\ \sin (\theta)\end{array}\right]$, with $\theta=0,0.6,1.2,1.8,2.4,3$, and solve (50). We obtain 6 contractively invariant ellipsoids, as plotted in Fig. 13 (the dotted curves). By Theorem 7, the convex hull of these ellipsoids (plotted in solid curve) is also an invariant set inside the domain of attraction. The inner ellipsoid in dashed curve is the largest invariant ellipsoid $\mathcal{E}\left(P, \rho_{\max }\right)$ in Example 1. It is much smaller than any of the six ellipsoids in dotted curves. This shows that the conservatism can be much reduced by taking $P$ as an optimization parameter. The outermost curve is the boundary of the actual domain of attraction obtained 


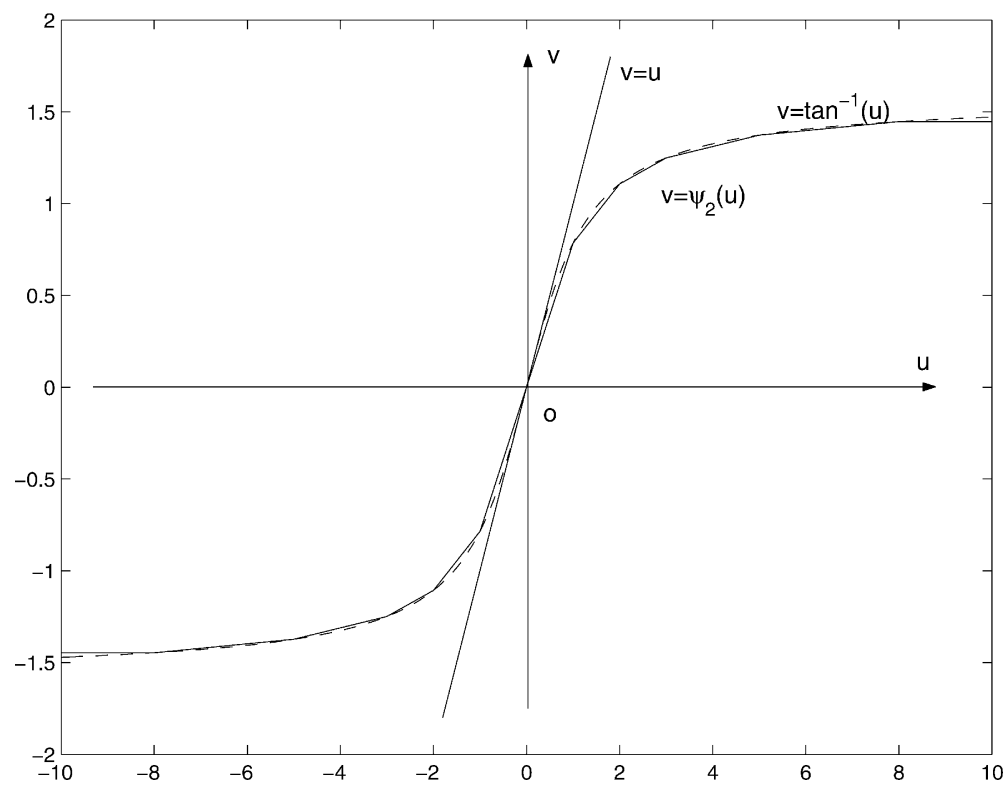

Fig. 12. Approximation of the function $\tan ^{-1}(u)$.

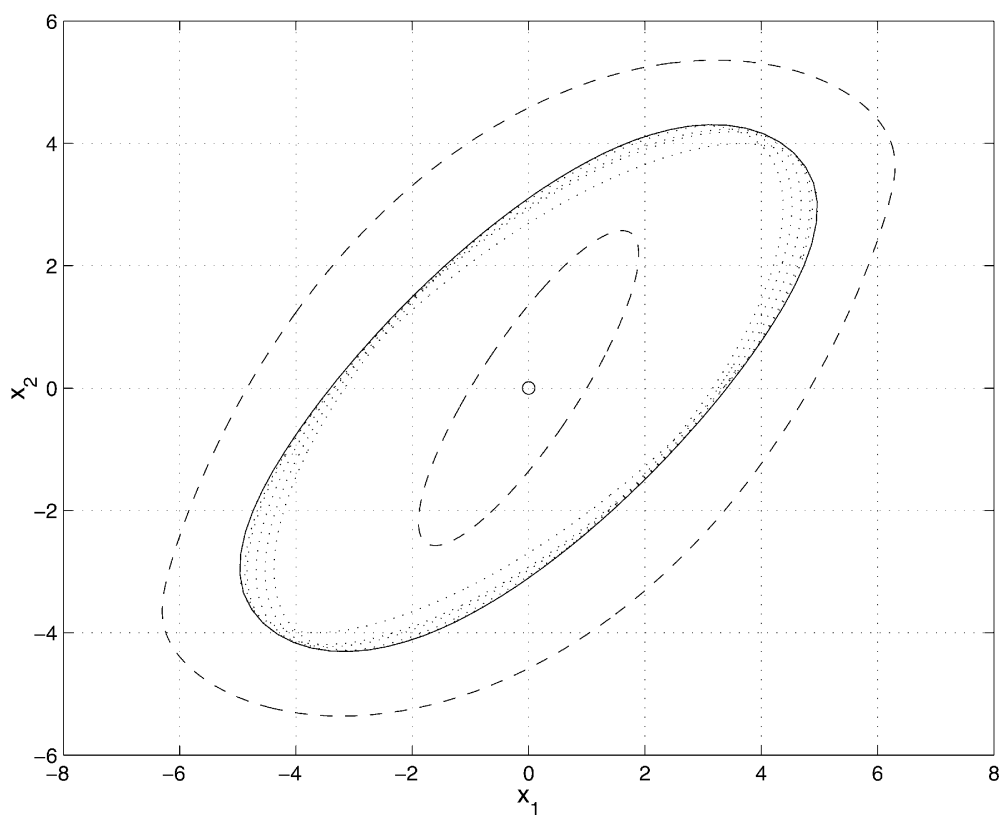

Fig. 13. Estimation of the domain of attraction.

by numerical method. It appears that there is still some space for the ellipsoids to expand such that they are still inside the domain of attraction. However, if they grow any larger, they will no longer be contractively invariant, not even invariant. This exhibits the difference between the quadratic stability region and the asymptotic stability region.

\section{CONCLUSION}

This paper presents a complete characterization of absolutely contractively invariant ellipsoids for systems with one nonlinear component which satisfies a generalized sector condition. LMI optimization techniques are proposed for maximizing the absolutely contractively invariant ellipsoids with respect to some shape reference set. It is also shown that the convex hull of a set of absolutely contractively invariant ellipsoids is also absolutely contractively invariant and hence can be used as an estimate of the domain of attraction. It should be noted that all the results in this paper are developed for systems with a single nonlinear component. Future effort will be made to extend these results to systems with multiple nonlinear components. Although in that case, the necessity of the corresponding LMI conditions for set invariance may be lost.

\section{REFERENCES}

[1] M. A. Aizerman and F. R. Gantmacher, Absolute Stability of Regulator Systems. San Francisco, CA: Holden-Day, 1964.

[2] M. Arcak and A. Teel, "Input-to-state stability for a class of lurie systems," Automatica, vol. 38, pp. 1945-1949, 2002. 
[3] D. S. Bernstein and A. N. Michel, "A chronological bibliography on saturating actuators," Int. J. Robust Nonlin. Control, vol. 5, pp. 375-380, 1995.

[4] S. Boyd, L. El Ghaoui, E. Feron, and V. Balakrishnan, Linear Matrix Inequalities in Systems and Control Theory, ser. SIAM Studies in Applied Mathematics. Philadelphia: SIAM, 1994.

[5] Y.-S. Chou, A. L. Tits, and V. Balakrishnan, "Stability multipliers and $\mu$ upper bounds: Connections and implications for numerical verification of frequency domain conditions," IEEE Trans. Automat. Contr., vol. 44, pp. 906-913, June 1999.

[6] T. Chu, L. Huang, and L. Wang, "Absolute stability and guaranteed domain of attraction for MIMO discrete-time Lur'e systems," in Proc. 40th IEEE Conf. Decision Control, Orlando, FL, 2001, pp. 1711-1716.

[7] E. J. Davison and E. M. Kurak, "A computational method for determining quadratic lyapunov functions for nonlinear systems," Automatica, vol. 7, pp. 627-636, 1971

[8] E. G. Gilbert and K. T. Tan, "Linear systems with state and control constraints: The theory and application of maximal output admissible sets," IEEE Trans. Automat. Contr., vol. 36, pp. 1008-1020, Sept. 1991.

[9] K. Gu, "Absolute stability of systems under block diagonal memoryless uncertainties," Automatica, vol. 31, no. 4, pp. 581-584, 1995.

[10] W. M. Haddad, V. Kapila, and V. S. Chellabonia, "Guaranteed domain of attraction for multivariable lur'e systems via open lyapunov surfaces," in Proc. 13th IFAC Wold Congr., San Francisco, CA, 1996, pp. 13-18.

[11] H. Hindi and S. Boyd, "Analysis of linear systems with saturating using convex optimization," in Proc. 37th IEEE Conf. Decision Control, 1998, pp. 903-908.

[12] J. C. Hsu and A. U. Meyer, Modern Control Principles and Applications. New York: McGraw-Hill, 1968.

[13] T. Hu and Z. Lin, Control Systems with Actuator Saturation: Analysis and Design. Boston, MA: Birkhäuser, 2001.

[14] _ _ "Exact characterization of invariant ellipsoids for linear systems with saturating actuators," IEEE Trans. Automat. Contr., vol. 47, pp. 164-169, Jan. 2002.

[15] — - "Composite quadratic lyapunov functions for constrained control systems," IEEE Trans. Automat. Contr., vol. 48, pp. 440-450, Mar. 2003.

[16] T. Hu, Z. Lin, and B. M. Chen, "An analysis and design method for linear systems subject to actuator saturation and disturbance," Automatica, vol. 38, no. 2, pp. 351-359, 2002.

[17] T. Hu, Z. Lin, and L. Qiu, "Stabilization of exponentially unstable linear systems with saturating actuators," IEEE Trans. Automat. Contr., vol. 46, pp. 973-979, June 2001.

[18] H. Khalil, Nonlinear Systems. Upper Saddle River, NJ: Prentice-Hall, 1996.

[19] T. Kiyama and T. Iwasaki, "On the use of multi-loop circle for saturating control synthesis," Syst. Control Lett., vol. 41, pp. 105-114, 2000

[20] D. Liu and A. N. Michel, Dynamical Systems With Saturation Nonlinearities. London, U.K.: Springer-Verlag, 1994, vol. 195, Lecture Notes in Control and Information Sciences.

[21] D. Liu and A. Molchanov, "Criteria for robust absolute stability of timevarying nonlinear continuous-time systems," Automatica, vol. 38, pp. 627-637, 2002

[22] A. Molchanov and D. Liu, "Robust absolute stability of time-varying nonlinear discrete-time systems," IEEE Trans. Circuit Syst. I, vol. 49, pp. 1129-1137, Aug. 2002.

[23] K. A. Loparo and G. L. Blankenship, "Estimating the domain of attraction of nonlinear feedback systems," IEEE Trans. Automat. Contr., vol. AC-23, pp. 602-607, Aug. 1978.

[24] K. S. Narendra and J. Taylor, Frequency Domain Methods for Absolute Stability. New York: Academic, 1973.

[25] A. Megretski and A. Rantzer, "System analysis via integral quadratic constraints," IEEE Trans. Automat. Contr., vol. 42, pp. 819-830, May 1997.

[26] J-J. E. Slotine and W. Li, Applied Nonlinear Control. Upper Saddle River, NJ: Prentice-Hall, 1991.

[27] M. M. Vidyasagar, Nonlinear Systems Analysis. Upper Saddle River, NJ: Prentice-Hall, 1993.

[28] T. Wada, M. Ikeda, Y. Ohta, and D. D. Siljak, "Parametric absolute stability of lur'e systems," IEEE Trans. Automat. Contr., vol. 43, pp. 1649-1653, Nov. 1998

[29] J. A. Walker and N. H. McClamroch, "Finite regions of attraction for the problem of lur'e,” Int. J. Control, vol. 6, no. 4, pp. 331-336, 1967.
[30] S. Weissenberger, "Application of results from the absolute stability to the computation of finite stability domains," IEEE Trans. Automat. Contr., vol. AC-13, pp. 124-125, Jan. 1968.

[31] J. L. Willems, "The computation of finte stability regions by means of open Lyapunov surfaces," Int. J. Control, vol. 10, no. 5, pp. 537-544, 1969.

[32] V. A. Yakubovich, "Frequency conditions for the absolute stability of control systems with several nonlinear and linear nonstationary blocks," Automat. Rem. Control, vol. 28, pp. 857-880, 1967.

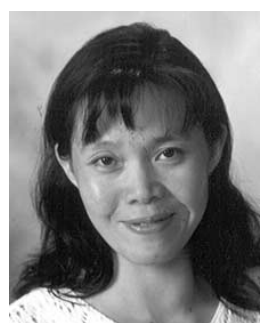

Tingshu Hu (S'99-SM'01) received the B.S. and M.S. degrees in electrical engineering from Shanghai Jiao Tong University, China, in 1985 and 1988, respectively, and the Ph.D. degree in electrical engineering from University of Virginia, Charlottesville, in 2001.

She is currently a Research Associate with the Department of Electrical and Computer Engineering, University of Virginia. Her research interests include nonlinear systems theory, optimization, robust control theory and control application in mechatronic systems, and biomechanical systems. She is a coauthor (with Z. Lin) of the book Control Systems with Actuator Saturation: Analysis and Design (Boston, MA: Birkhäuser, 2001).

Dr. Hu is currently an Associate Editor on the Conference Editorial Board of the IEEE Control Systems Society.

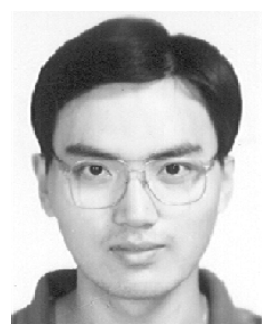

Bin Huang (S'00) received the B.S. degree in electrical engineering from the University of Science and Technology of China, Beijing, and the M.S. degree in electrical engineering from National University of Singapore, in 1998 and 2001, respectively. He is currentlly working toward the Ph.D. degree in the Department of Electrical and Computer Engineering, University of Virginia, Charlottesville.

His current research interests include real-time control and nonlinear control.

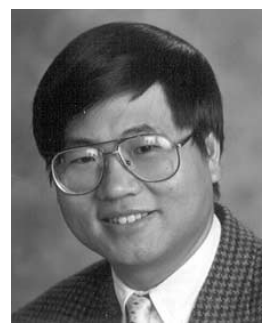

Zongli Lin (S'89-M'90-SM'98) received the B.S. degree in mathematics and computer science from Xiamen University, Xiamen, China, in 1983, the M.E. degree in automatic control from the Chinese Academy of Space Technology, Beijing, China, in 1989, and the Ph.D. degree in electrical and computer engineering from Washington State University, Pullman, in 1994.

$\mathrm{He}$ is currently an Associate Professor with the Department of Electrical and Computer Engineering at University of Virginia, Charlottesville. Previously, he has worked as a Control Engineer at the Chinese Academy of Space Technology and as an Assistant Professor with the Department of Applied Mathematics and Statistics at State University of New York at Stony Brook. His current research interest include nonlinear control, robust control, and modeling and control of magnetic bearing system. In these areas, he has published several papers. He is also the author of Low Gain Feedback (London, U.K.: Springer-Verlag, 1998) and a coauthor (with T. Hu) of Control Systems with Actuator Saturation: Analysis and Design (Birkhäuser, Boston, 2001).

Dr. Lin served as an Associate Editor of IEEE TRANSACTIONS ON AUTOMATIC CONTROL from 2001 to 2003. He is currently a member of the IEEE Control Systems Society's Technical Committee on Nonlinear Systems and Control, and heads its Working Group on Control with Constraints. He was the recipient of a U.S. Office of Naval Research Young Investigator Award. 